OPEN $\curvearrowright$ ACCESS COMMUNICATIONS

ISSN 2056-9890

\section{Crystal structure of tris[ $\mu_{2}$-bis(diphenyl- phosphanyl)methane- $\left.\kappa^{2} P: P^{\prime}\right] \mathrm{di}-\mu_{3}$-iodido- trisilver(I) iodide- $N$-phenylthiourea (1/1)}

Yupa Wattanakanjana, ${ }^{\mathrm{a} *}$ Arunpatcha Nimthong-Roldán, ${ }^{\mathrm{b}}$ Suthida Palavat ${ }^{\mathrm{a}}$ and Walailak Puetpaiboon ${ }^{\mathrm{a}}$

a Department of Chemistry, Faculty of Science, Prince of Songkla University, Hat Yai, Songkhla 90112, Thailand, and ${ }^{\mathbf{b}}$ Department of Chemistry, Youngstown State University, 1 University Plaza, 44555, Youngstown, OH, USA. *Correspondence e-mail: yupa.t@psu.ac.th

Received 2 September 2015; accepted 12 September 2015

Edited by A. J. Lough, University of Toronto, Canada

The title complex, $\left[\mathrm{Ag}_{3} \mathrm{I}_{2}\left(\mathrm{C}_{25} \mathrm{H}_{22} \mathrm{P}_{2}\right)_{3}\right] \mathrm{I} \cdot \mathrm{C}_{7} \mathrm{H}_{8} \mathrm{~N}_{2} \mathrm{~S}$, comprises a trinuclear $\left[\mathrm{Ag}_{3} \mathrm{I}_{2}\left(\mathrm{C}_{25} \mathrm{H}_{22} \mathrm{P}_{2}\right)_{3}\right]^{+}$unit, an $\mathrm{I}^{-}$anion and one $N, N^{\prime}-$ phenylthiourea molecule (ptu). Two $\mu_{3}$-bridging $\mathrm{I}^{-}$anions are linked by three $\mathrm{Ag}^{\mathrm{I}}$ ions, leading to the formation of a dicapped triangular motif with $\mathrm{Ag} \cdots \mathrm{Ag}$ separations in the range 3.0823 (5)-3.2999 (5) $\AA$. Each $\mathrm{Ag}^{\mathrm{I}}$ atom exhibits a distorted tetrahedral geometry, with coordination to two I atoms and two $\mathrm{P}$ atoms from bis(diphenylphosphanyl)methane ligands. In the crystal, the $\mathrm{I}^{-}$anion is linked to the ptu molecule through two $\mathrm{N}-\mathrm{H} \cdots \mathrm{I}$ hydrogen bonds [graph-set motif $R_{2}^{1}(6)$ ]. These $\mathrm{N}-\mathrm{H} \cdots \mathrm{I}$ hydrogen bonds, in addition to weak $\mathrm{C}-\mathrm{H} \cdots \mathrm{S}$ and $\mathrm{C}-\mathrm{H} \cdots \mathrm{I}$ hydrogen bonds, form zigzag chains along [010]. Two of the phenyl rings of two dppm ligands are disordered over two sets of sites with refined occupancies of 0.557 (16) and 0.443 (16).

Keywords: crystal structure; $N, N^{\prime}$-phenylthiourea; silver complex; hydrogen bonding.

CCDC reference: 1424053

\section{Related literature}

For bis(diphenylphosphino)methane (dppm) complexes formed with metal(I) ions, see: Bera et al. (1998); Matsumoto et al. (2001); Nicola et al. (2005). For the complex $\left[\mathrm{Ag}_{3}\left(\mathrm{C}_{25} \mathrm{H}_{22} \mathrm{P}_{2}\right)_{3}\left(\mu_{3}-\mathrm{Br}\right)_{2}\right]^{+}$, see: Nimthong-Roldán et al. (2015).
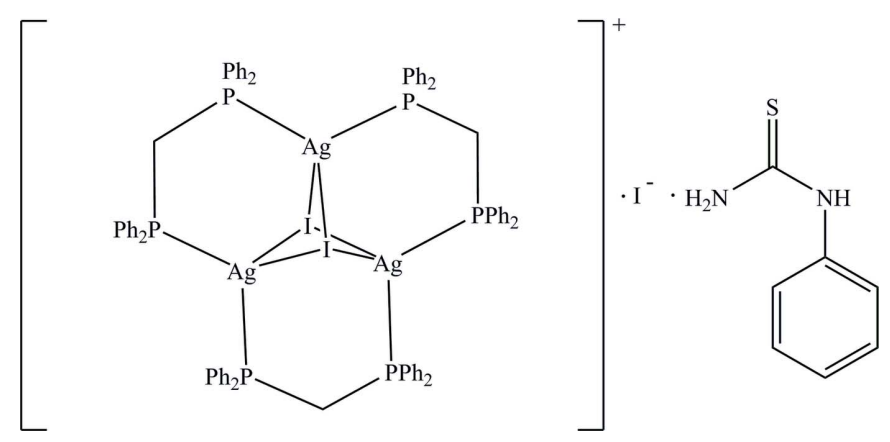

2. Experimental

2.1. Crystal data

$\left[\mathrm{Ag}_{3} \mathrm{I}_{2}\left(\mathrm{C}_{25} \mathrm{H}_{22} \mathrm{P}_{2}\right)_{3}\right] \mathrm{I} \cdot \mathrm{C}_{7} \mathrm{H}_{8} \mathrm{~N}_{2} \mathrm{~S}$

$M_{r}=2009.62$

Monoclinic, $P 2_{1} / n$

$a=10.8177$ (4) ^

$b=28.5680(11) \AA$

$c=26.1639$ (11) $\AA$

$\beta=95.315(2)^{\circ}$

$V=8050.9(5) \AA^{3}$

$Z=4$

$\mathrm{Cu} K \alpha$ radiation

$\mu=16.53 \mathrm{~mm}^{-1}$

$T=296 \mathrm{~K}$

$0.14 \times 0.04 \times 0.03 \mathrm{~mm}$

\subsection{Data collection}

Bruker Prospector CCD diffractometer

Absorption correction: multi-scan (SADABS; Bruker, 2013)

$T_{\min }=0.394, T_{\max }=0.753$

82145 measured reflections 14160 independent reflections 12277 reflections with $I>2 \sigma(I)$ $R_{\text {int }}=0.056$

\subsection{Refinement}

$R\left[F^{2}>2 \sigma\left(F^{2}\right)\right]=0.039$

$w R\left(F^{2}\right)=0.104$

$S=1.08$

14160 reflections

983 parameters

216 restraints

$\mathrm{H}$-atom parameters constrained

$\Delta \rho_{\max }=1.16{\mathrm{e} \AA^{-3}}^{-3}$

$\Delta \rho_{\text {min }}=-1.04{\mathrm{e} \AA^{-3}}^{-3}$

Table 1

Hydrogen-bond geometry $\left(\AA{ }^{\circ}\right)$.

\begin{tabular}{lllll}
\hline$D-\mathrm{H} \cdots A$ & $D-\mathrm{H}$ & $\mathrm{H} \cdots A$ & $D \cdots A$ & $D-\mathrm{H} \cdots A$ \\
\hline $\mathrm{N} 1-\mathrm{H} 1 A \cdots \mathrm{I} 3$ & 0.86 & 2.82 & $3.633(9)$ & 159 \\
$\mathrm{~N} 2-\mathrm{H} 2 A \cdots \mathrm{I} 3$ & 0.86 & 2.72 & $3.568(6)$ & 170 \\
$\mathrm{C} 53-\mathrm{H} 53 \cdots \mathrm{S} 1^{\mathrm{i}}$ & 0.99 & 2.88 & $3.67(2)$ & 143 \\
$\mathrm{C} 13-\mathrm{H} 13 B \cdots \mathrm{I} 3$ & 0.99 & 3.04 & $3.933(4)$ & 153 \\
\hline
\end{tabular}

Symmetry code: (i) $-x+\frac{3}{2}, y-\frac{1}{2},-z+\frac{3}{2}$.

Data collection: APEX2 (Bruker, 2013); cell refinement: SAINT (Bruker, 2013); data reduction: $S A I N T$; $\operatorname{program}(\mathrm{s})$ used to solve structure: SHELXS97 (Sheldrick, 2008); program(s) used to refine structure: SHELXL2014 (Sheldrick, 2015) and SHELXLE (Hübschle et al., 2011); molecular graphics: Mercury (Macrae et al., 2008); software used to prepare material for publication: publCIF (Westrip, 2010).

\title{
Acknowledgements
}

We are grateful to Department of Chemistry, Faculty of Science, Prince of Songkla University, for financial assistance. 
We would like to thank Youngstown State University for access to their X-ray diffraction facility, funded in part by NSF Grant DMR 1337296.

Supporting information for this paper is available from the IUCr electronic archives (Reference: LH5785).

\section{References}

Bera, J. K., Nethaji, M. \& Samuelson, A. G. (1998). Inorg. Chem. 38, 218-228. Bruker (2013). APEX2, SAINT and SADABS . Bruker AXS Inc., Madison, Wisconsin, USA.
Hübschle, C. B., Sheldrick, G. M. \& Dittrich, B. (2011). J. Appl. Cryst. 44, 12811284

Macrae, C. F., Bruno, I. J., Chisholm, J. A., Edgington, P. R., McCabe, P., Pidcock, E., Rodriguez-Monge, L., Taylor, R., van de Streek, J. \& Wood, P. A. (2008). J. Appl. Cryst. 41, 466-470.

Matsumoto, K., Tanaka, R., Shimomura, R., Matsumoto, C. \& Nakao, Y. (2001). Inorg. Chim. Acta, 322, 125-129.

Nicola, C. D., Effendy, Fazaroh, F., Pettinari, C., Skelton, B. W., Somers, N. \& White, A. H. (2005). Inorg. Chim. Acta, 358, 720-734.

Nimthong-Roldán, A., Wattanakanjana, Y. \& Rodkeaw, J. (2015). Acta Cryst. E71, m89-m90.

Sheldrick, G. M. (2008). Acta Cryst. A64, 112-122.

Sheldrick, G. M. (2015). Acta Cryst. C71, 3-8.

Westrip, S. P. (2010). J. Appl. Cryst. 43, 920-925. 


\title{
supporting information
}

Acta Cryst. (2015). E71, m187-m188 [https://doi.org/10.1107/S2056989015017120]

\section{Crystal structure of tris $\left[\mu_{2}\right.$-bis(diphenylphosphanyl)methane- $\left.\kappa^{2} P: P^{\prime}\right] d i-\mu_{3}$-iodido- trisilver(I) iodide- $N$-phenylthiourea (1/1)}

\author{
Yupa Wattanakanjana, Arunpatcha Nimthong-Roldán, Suthida Palavat and Walailak
}

\section{Puetpaiboon}

\section{S1. Comment}

Complexes of bis(diphenylphosphino)methane (dppm) with metal(I) ions have been extensively studied (Bera et al. 1998; Matsumoto et al. 2001; Nicola et al. 2005). Recently, we reported the complex which we prepared by reacting silver (I) bromide and dppm, followed by the addition of $N, N^{\prime}$-phenylthiourea (ptu) in acetonitrile solvent. An unexpected complex $\left[\mathrm{Ag}_{3}\left(\mathrm{C}_{25} \mathrm{H}_{22} \mathrm{P}_{2}\right)_{3}\left(\mu_{3}-\mathrm{Br}\right)_{2}\right]^{+}$unit was formed with uncoordinated ptu (Nimthong-Roldán et al. 2015). Herein, we present the complex formed using silver(I) iodide instead of silver(I) bromide under the same conditions.

The title complex consists of a trinuclear $\left[\mathrm{Ag}_{3}\left(\mathrm{C}_{25} \mathrm{H}_{22} \mathrm{P}_{2}\right)_{3}\left(\mu_{3}-\mathrm{I}\right)_{2}\right]^{+}$unit, a discrete $\mathrm{I}^{-}$anion and one $N, N^{\prime}$-phenylthiourea molecule (ptu). Three $\mathrm{Ag}^{\mathrm{I}}$ ions in the corners of the trianglular plane are linked via two bridging $\mu_{3}-\mathrm{I}$ anions, forming a dicapped triangular geometry with a Ag*.Ag separations range of 3.0823 (5)-3.2999 (5) Å. Each $\mathrm{Ag}^{\mathrm{I}}$ ion coordinates to two iodide ions and two $\mathrm{P}$ atoms of two dppm ligands in a distorted tetrahedral geometry (Fig 1). In the crystal, two N$\mathrm{H} \cdots \mathrm{I}$ hydrogen bonds exist between the iodide anion and ptu molecule [graph-set motif $\mathrm{R}^{2}{ }_{1}(6)$ ] (Table 1). These hydrogen bonds are in turn connected via weak $\mathrm{C}-\mathrm{H} \cdots \mathrm{S}$ and $\mathrm{C}-\mathrm{H} \cdots \mathrm{I}$ hydrogen bonds, forming a zig-zag chain along [010] (Fig. 2, Table 1). Two of the phenyl rings from two dppm ligands are disordered over two sites with refined occupancies 0.557 (16) and $0.443(16)$.

\section{S2. Synthesis and crystallization}

Bis(diphenylphosphino)methane, dppm, $(0.1 \mathrm{~g}, 0.26 \mathrm{mmol})$ was dissolved in $30 \mathrm{ml}$ of acetonitrile at $343 \mathrm{~K}$ and then silver(I) iodide, AgI, (0.06 g, $0.26 \mathrm{mmol})$ was added. The mixture was stirred for $4 \mathrm{hr}$ and then $N, N^{\prime}$-phenylthiourea, ptu, $(0.04 \mathrm{~g}, 0.26 \mathrm{mmol})$ was added and the new reaction mixture was heated under reflux for $6 \mathrm{hr}$ during which the precipitate gradually disappeared. The resulting clear solution was filtered and left to evaporate at room temperature. The crystalline complex, which deposited upon standing for several days, was filtered off and dried in vacuo.

\section{S3. Refinement details}

$\mathrm{H}$ atoms bonded to $\mathrm{C}$ and $\mathrm{N}$ atoms were included in calculated positions and were refined with a riding model using distances of $0.95 \AA(\operatorname{aryl~H})$, and $U_{\text {iso }}(\mathrm{H})=1.2 U_{\text {eq }}(\mathrm{C}) ; 0.99 \AA\left(\mathrm{CH}_{2}\right)$ and $U_{\text {iso }}(\mathrm{H})=1.5 U_{\text {eq }}(\mathrm{C}) ; 0.88 \AA(\mathrm{NH})$, and $U_{\text {iso }}(\mathrm{H})=$ $1.2 U_{\text {eq }}(\mathrm{N})$. Two phenyl rings from two dppm ligands are disordered. The geometry of the minor component of each pair of disordered phenyl rings was restrained to be similar to that of the major componnet (within a standard deviation of $0.02 \AA$ ). Carbon atoms of one phenyl ring were restrained with effective standard deviation 0.01 to have the same Uij components. To ensure satisfactory refinement the atoms of each disorder component of the phenyl rings were restrained to lie within a common plane. The overall ratio of the two components of disorder, refined with the same free variable, is 0.557 (16):0.443 (16). 


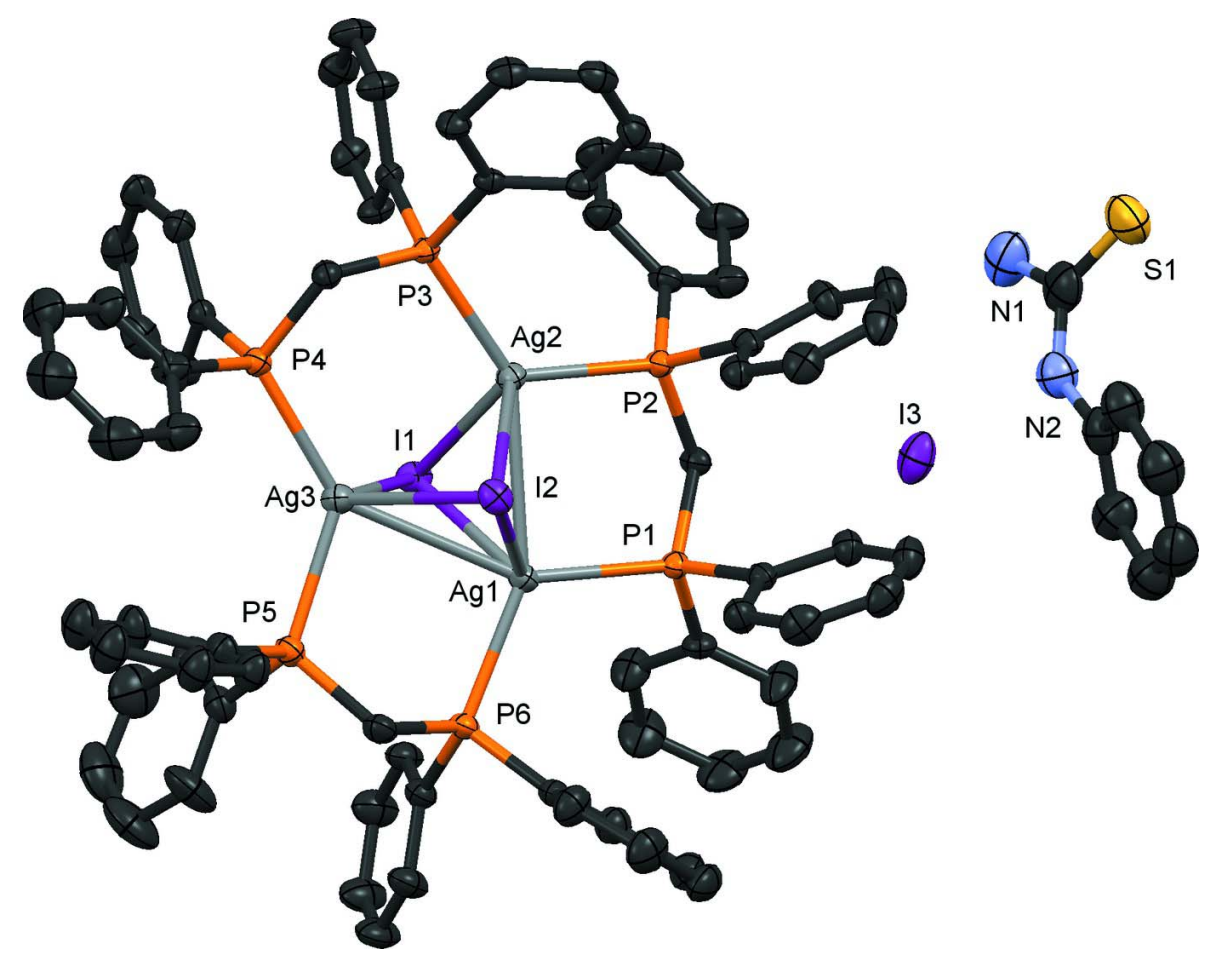

Figure 1

The molecular structure with displacement ellipsoids drawn at the 50\% probability level. All $\mathrm{H}$ atoms and the minor component of disorder are omitted for clarity.

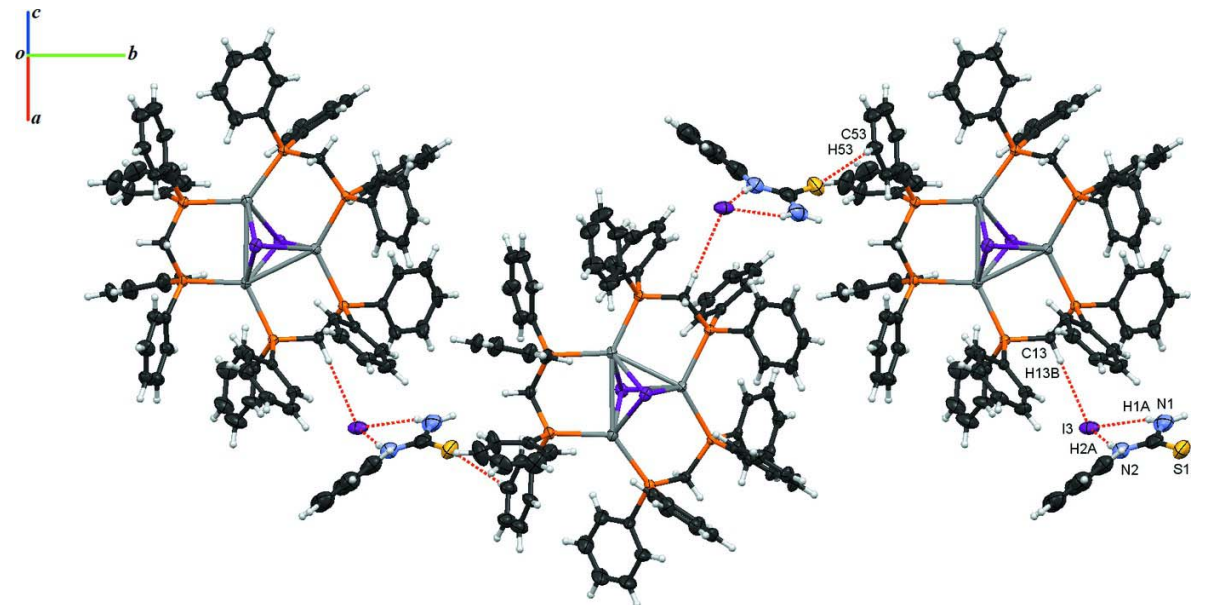

\section{Figure 2}

Part of the crystal structure showing intermolecular $\mathrm{N}-\mathrm{H} \cdots \mathrm{I}, \mathrm{C}-\mathrm{H} \cdots \mathrm{S}$ and $\mathrm{C}-\mathrm{H} \cdots \mathrm{I}$ hydrogen bonds as dashed lines, forming a chain along [010].

$\operatorname{Tris}\left[\mu_{2}\right.$-bis(diphenylphosphanyl)methane- $\left.\kappa^{2} P: P^{\prime}\right]$ di- $\mu_{3}$-iodido-trisilver(I) iodide- $N$-phenylthiourea (1/1)

Crystal data

$\left[\mathrm{Ag}_{3} \mathrm{I}_{2}\left(\mathrm{C}_{25} \mathrm{H}_{22} \mathrm{P}_{2}\right)_{3}\right] \mathrm{I} \cdot \mathrm{C}_{7} \mathrm{H}_{8} \mathrm{~N}_{2} \mathrm{~S}$

$M_{r}=2009.62$

Monoclinic, $P 2_{1} / n$

$$
\begin{aligned}
& a=10.8177(4) \AA \\
& b=28.5680(11) \AA \\
& c=26.1639(11) \AA
\end{aligned}
$$


$\beta=95.315(2)^{\circ}$

$V=8050.9(5) \AA^{3}$

$Z=4$

$F(000)=3944$

$D_{\mathrm{x}}=1.658 \mathrm{Mg} \mathrm{m}^{-3}$

$\mathrm{Cu} K \alpha$ radiation, $\lambda=1.54178 \AA$

Data collection

Bruker Prospector CCD diffractometer

Radiation source: I-mu-S microsource X-ray tube

Laterally graded multilayer (Goebel) mirror monochromator

$\omega$ and $\varphi$ scans

Absorption correction: multi-scan

(SADABS; Bruker, 2013)

\section{Refinement}

Refinement on $F^{2}$

Least-squares matrix: full

$R\left[F^{2}>2 \sigma\left(F^{2}\right)\right]=0.039$

$w R\left(F^{2}\right)=0.104$

$S=1.08$

14160 reflections

983 parameters

216 restraints

Primary atom site location: structure-invariant direct methods
Cell parameters from 9031 reflections

$\theta=2.3-66.5^{\circ}$

$\mu=16.53 \mathrm{~mm}^{-1}$

$T=296 \mathrm{~K}$

Block, colourless

$0.14 \times 0.04 \times 0.03 \mathrm{~mm}$

$T_{\min }=0.394, T_{\max }=0.753$

82145 measured reflections

14160 independent reflections

12277 reflections with $I>2 \sigma(I)$

$R_{\text {int }}=0.056$

$\theta_{\text {max }}=67.2^{\circ}, \theta_{\min }=2.3^{\circ}$

$h=-12 \rightarrow 12$

$k=-34 \rightarrow 33$

$l=-30 \rightarrow 31$

Secondary atom site location: difference Fourier map

Hydrogen site location: inferred from neighbouring sites

$\mathrm{H}$-atom parameters constrained

$w=1 /\left[\sigma^{2}\left(F_{\mathrm{o}}^{2}\right)+(0.0506 P)^{2}+12.1838 P\right]$

where $P=\left(F_{\mathrm{o}}^{2}+2 F_{\mathrm{c}}^{2}\right) / 3$

$(\Delta / \sigma)_{\max }=0.002$

$\Delta \rho_{\max }=1.16 \mathrm{e} \AA^{-3}$

$\Delta \rho_{\min }=-1.04 \mathrm{e} \AA^{-3}$

Special details

Geometry. All e.s.d.'s (except the e.s.d. in the dihedral angle between two 1.s. planes) are estimated using the full covariance matrix. The cell e.s.d.'s are taken into account individually in the estimation of e.s.d.'s in distances, angles and torsion angles; correlations between e.s.d.'s in cell parameters are only used when they are defined by crystal symmetry. An approximate (isotropic) treatment of cell e.s.d.'s is used for estimating e.s.d.'s involving l.s. planes.

Fractional atomic coordinates and isotropic or equivalent isotropic displacement parameters $\left(\AA^{2}\right)$

\begin{tabular}{llllll}
\hline & $x$ & $y$ & $z$ & $U_{\text {iso }}^{*} / U_{\text {eq }}$ & Occ. $(<1)$ \\
\hline I1 & $0.09573(3)$ & $0.07662(2)$ & $0.74036(2)$ & $0.04248(9)$ & \\
I2 & $0.49507(3)$ & $0.04371(2)$ & $0.81433(2)$ & $0.04443(9)$ & \\
I3 & $0.39502(4)$ & $0.18325(2)$ & $0.50190(2)$ & $0.07625(14)$ & \\
Ag1 & $0.32400(3)$ & $0.03008(2)$ & $0.71995(2)$ & $0.04176(9)$ & \\
Ag2 & $0.33079(3)$ & $0.12525(2)$ & $0.77564(2)$ & $0.03938(9)$ & \\
Ag3 & $0.23020(4)$ & $0.03137(2)$ & $0.83250(2)$ & $0.04526(10)$ & \\
S1 & $0.8130(3)$ & $0.30895(9)$ & $0.55157(10)$ & $0.1102(8)$ & \\
N1 & $0.5783(8)$ & $0.2843(3)$ & $0.5464(3)$ & $0.106(2)$ & \\
H1A & 0.5193 & 0.2643 & 0.5407 & $0.127^{*}$ & \\
H1B & 0.5620 & 0.3126 & 0.5550 & $0.127^{*}$ & \\
N2 & $0.7041(6)$ & $0.2262(2)$ & $0.5281(2)$ & $0.0783(16)$ & \\
H2A & 0.6339 & 0.2121 & 0.5223 & $0.094^{*}$ & \\
P1 & $0.39878(11)$ & $0.06755(4)$ & $0.64386(4)$ & $0.0340(2)$ &
\end{tabular}




\begin{tabular}{|c|c|c|c|c|}
\hline $\mathrm{C} 1$ & $0.3240(5)$ & $0.04256(16)$ & $0.58416(18)$ & $0.0409(11)$ \\
\hline $\mathrm{C} 2$ & $0.2052(6)$ & 0.0260 & $0.5856(2)$ & $0.0676(17)$ \\
\hline $\mathrm{H} 2$ & 0.1655 & 0.0292 & 0.6154 & $0.081^{*}$ \\
\hline $\mathrm{C} 3$ & $0.1443(7)$ & 0.0045 & $0.5432(3)$ & $0.088(2)$ \\
\hline $\mathrm{H} 3$ & 0.0644 & -0.0071 & 0.5447 & $0.106^{*}$ \\
\hline $\mathrm{C} 4$ & $0.2018(8)$ & 0.0004 & $0.4990(3)$ & $0.086(2)$ \\
\hline $\mathrm{H} 4$ & 0.1605 & -0.0134 & 0.4701 & $0.103^{*}$ \\
\hline $\mathrm{C} 5$ & $0.3164(8)$ & 0.0163 & $0.4975(3)$ & 0.099 (3) \\
\hline H5 & 0.3560 & 0.0126 & 0.4677 & $0.119^{*}$ \\
\hline C6 & $0.3785(7)$ & 0.0384 & $0.5396(2)$ & $0.080(2)$ \\
\hline H6 & 0.4577 & 0.0504 & 0.5374 & $0.096^{*}$ \\
\hline $\mathrm{C} 7$ & $0.5644(4)$ & 0.06579 (16) & $0.63783(17)$ & $0.0374(10)$ \\
\hline $\mathrm{C} 8$ & $0.6341(5)$ & 0.03319 (19) & $0.6672(2)$ & $0.0512(12)$ \\
\hline $\mathrm{H} 8$ & 0.5946 & 0.0128 & 0.6881 & $0.061 *$ \\
\hline C9 & $0.7616(6)$ & $0.0304(2)$ & $0.6660(3)$ & $0.0658(16)$ \\
\hline H9 & 0.8076 & 0.0086 & 0.6861 & $0.079 *$ \\
\hline $\mathrm{C} 10$ & $0.8188(6)$ & $0.0600(3)$ & $0.6348(2)$ & $0.0640(16)$ \\
\hline $\mathrm{H} 10$ & 0.9044 & 0.0583 & 0.6339 & $0.077^{*}$ \\
\hline $\mathrm{C} 11$ & $0.7528(5)$ & $0.0922(2)$ & $0.6048(2)$ & $0.0593(15)$ \\
\hline H11 & 0.7934 & 0.1119 & 0.5835 & $0.071 *$ \\
\hline $\mathrm{C} 12$ & $0.6258(5)$ & $0.0954(2)$ & $0.6062(2)$ & $0.0484(12)$ \\
\hline H12 & 0.5809 & 0.1175 & 0.5859 & $0.058^{*}$ \\
\hline $\mathrm{C} 13$ & $0.3510(5)$ & $0.12916(15)$ & $0.63727(17)$ & $0.0366(10)$ \\
\hline H13A & 0.2622 & 0.1304 & 0.6277 & $0.044 *$ \\
\hline H13B & 0.3920 & 0.1432 & 0.6096 & $0.044 *$ \\
\hline $\mathrm{P} 2$ & $0.38689(11)$ & 0.16385 (4) & $0.69593(4)$ & $0.0341(2)$ \\
\hline $\mathrm{C} 14$ & $0.5505(5)$ & $0.17912(16)$ & $0.69543(17)$ & $0.0401(10)$ \\
\hline $\mathrm{C} 15$ & $0.6363(5)$ & 0.15182 (19) & $0.72515(19)$ & $0.0478(12)$ \\
\hline H15 & 0.6090 & 0.1283 & 0.7459 & $0.057^{*}$ \\
\hline $\mathrm{C} 16$ & $0.7618(6)$ & $0.1597(2)$ & $0.7238(2)$ & $0.0628(15)$ \\
\hline H16 & 0.8187 & 0.1406 & 0.7428 & $0.075^{*}$ \\
\hline $\mathrm{C} 17$ & $0.8037(6)$ & $0.1952(3)$ & $0.6947(2)$ & $0.0705(19)$ \\
\hline H17 & 0.8884 & 0.2005 & 0.6941 & $0.085^{*}$ \\
\hline $\mathrm{C} 18$ & $0.7194(7)$ & $0.2231(3)$ & $0.6665(3)$ & $0.0731(19)$ \\
\hline H18 & 0.7474 & 0.2476 & 0.6472 & $0.088^{*}$ \\
\hline C19 & $0.5942(6)$ & $0.2152(2)$ & $0.6665(2)$ & $0.0564(14)$ \\
\hline H19 & 0.5382 & 0.2342 & 0.6470 & $0.068^{*}$ \\
\hline $\mathrm{C} 20$ & $0.2963(5)$ & $0.21695(16)$ & $0.68020(19)$ & $0.0426(11)$ \\
\hline $\mathrm{C} 21$ & $0.2735(7)$ & $0.2341(2)$ & $0.6309(2)$ & $0.0631(16)$ \\
\hline $\mathrm{H} 21$ & 0.3122 & 0.2203 & 0.6045 & $0.076^{*}$ \\
\hline $\mathrm{C} 22$ & $0.1944(8)$ & $0.2714(2)$ & $0.6202(3)$ & $0.077(2)$ \\
\hline $\mathrm{H} 22$ & 0.1810 & 0.2828 & 0.5869 & $0.093 *$ \\
\hline $\mathrm{C} 23$ & $0.1348(7)$ & $0.2918(2)$ & $0.6593(3)$ & $0.078(2)$ \\
\hline $\mathrm{H} 23$ & 0.0799 & 0.3165 & 0.6521 & $0.093^{*}$ \\
\hline $\mathrm{C} 24$ & $0.1568(7)$ & $0.2758(2)$ & $0.7079(3)$ & 0.0747 (19) \\
\hline $\mathrm{H} 24$ & 0.1167 & 0.2895 & 0.7340 & $0.090^{*}$ \\
\hline $\mathrm{C} 25$ & $0.2398(6)$ & $0.23871(19)$ & $0.7191(2)$ & $0.0596(15)$ \\
\hline $\mathrm{H} 25$ & 0.2568 & 0.2287 & 0.7529 & $0.072 *$ \\
\hline
\end{tabular}




\begin{tabular}{|c|c|c|c|c|c|}
\hline P3 & $0.31631(10)$ & $0.16684(4)$ & $0.85759(4)$ & $0.0306(2)$ & \\
\hline $\mathrm{C} 26$ & $0.4537(4)$ & $0.20236(15)$ & $0.87508(17)$ & $0.0341(9)$ & \\
\hline $\mathrm{C} 27$ & $0.5244(4)$ & $0.21633(17)$ & $0.83608(19)$ & $0.0413(10)$ & \\
\hline $\mathrm{H} 27$ & 0.5020 & 0.2067 & 0.8025 & $0.050^{*}$ & \\
\hline $\mathrm{C} 28$ & $0.6280(5)$ & $0.24443(19)$ & $0.8466(2)$ & $0.0520(13)$ & \\
\hline $\mathrm{H} 28$ & 0.6734 & 0.2540 & 0.8200 & $0.062 *$ & \\
\hline $\mathrm{C} 29$ & $0.6640(5)$ & $0.25822(18)$ & $0.8956(2)$ & $0.0529(13)$ & \\
\hline $\mathrm{H} 29$ & 0.7344 & 0.2767 & 0.9024 & $0.063^{*}$ & \\
\hline $\mathrm{C} 30$ & $0.5955(5)$ & $0.24460(19)$ & $0.9349(2)$ & $0.0521(13)$ & \\
\hline $\mathrm{H} 30$ & 0.6198 & 0.2542 & 0.9683 & $0.063^{*}$ & \\
\hline $\mathrm{C} 31$ & $0.4909(5)$ & $0.21680(18)$ & $0.9253(2)$ & $0.0451(11)$ & \\
\hline $\mathrm{H} 31$ & 0.4456 & 0.2078 & 0.9521 & $0.054^{*}$ & \\
\hline $\mathrm{C} 32$ & $0.1869(4)$ & $0.20864(15)$ & $0.85560(16)$ & $0.0333(9)$ & \\
\hline $\mathrm{C} 33$ & $0.1905(5)$ & $0.24916(19)$ & $0.8851(2)$ & $0.0536(13)$ & \\
\hline H33 & 0.2598 & 0.2557 & 0.9077 & $0.064^{*}$ & \\
\hline $\mathrm{C} 34$ & $0.0911(6)$ & $0.2796(2)$ & 0.8809 & $0.0660(17)$ & \\
\hline H34 & 0.0934 & 0.3066 & 0.9008 & $0.079 *$ & \\
\hline $\mathrm{C} 35$ & $-0.0121(5)$ & $0.2700(2)$ & $0.8472(2)$ & $0.0584(14)$ & \\
\hline H35 & -0.0788 & 0.2907 & 0.8442 & $0.070 *$ & \\
\hline $\mathrm{C} 36$ & $-0.0153(5)$ & $0.2304(2)$ & $0.8186(2)$ & $0.0546(13)$ & \\
\hline H36 & -0.0850 & 0.2238 & 0.7963 & $0.066^{*}$ & \\
\hline C37 & $0.0833(5)$ & $0.19986(18)$ & $0.82229(19)$ & $0.0449(11)$ & \\
\hline H37 & 0.0801 & 0.1730 & 0.8021 & $0.054 *$ & \\
\hline $\mathrm{C} 38$ & $0.3038(4)$ & 0.13077 (16) & $0.91539(17)$ & $0.0363(10)$ & \\
\hline $\mathrm{H} 38 \mathrm{~A}$ & 0.3851 & 0.1182 & 0.9266 & $0.044^{*}$ & \\
\hline $\mathrm{H} 38 \mathrm{~B}$ & 0.2788 & 0.1507 & 0.9426 & $0.044 *$ & \\
\hline P4 & $0.19276(11)$ & $0.08189(4)$ & $0.90601(4)$ & $0.0349(2)$ & \\
\hline C39 & $0.0385(4)$ & 0.10805 (16) & $0.90295(18)$ & $0.0381(10)$ & \\
\hline $\mathrm{C} 40$ & $0.0130(5)$ & 0.14851 (19) & $0.9295(2)$ & $0.0486(12)$ & \\
\hline $\mathrm{H} 40$ & 0.0757 & 0.1631 & 0.9504 & $0.058^{*}$ & \\
\hline $\mathrm{C} 41$ & $-0.1067(6)$ & $0.1673(2)$ & $0.9248(2)$ & $0.0588(15)$ & \\
\hline $\mathrm{H} 41$ & -0.1238 & 0.1946 & 0.9423 & $0.071^{*}$ & \\
\hline $\mathrm{C} 42$ & $-0.1978(6)$ & $0.1457(2)$ & 0.8945 & $0.0642(16)$ & \\
\hline $\mathrm{H} 42$ & -0.2774 & 0.1583 & 0.8913 & $0.077 *$ & \\
\hline $\mathrm{C} 43$ & $-0.1744(5)$ & $0.1052(2)$ & $0.8683(3)$ & $0.0646(16)$ & \\
\hline $\mathrm{H} 43$ & -0.2382 & 0.0903 & 0.8483 & $0.077^{*}$ & \\
\hline $\mathrm{C} 44$ & $-0.0551(5)$ & $0.0869(2)$ & $0.8721(2)$ & $0.0521(13)$ & \\
\hline $\mathrm{H} 44$ & -0.0383 & 0.0602 & 0.8536 & $0.062 *$ & \\
\hline $\mathrm{C} 45$ & $0.2138(18)$ & $0.0542(7)$ & $0.9702(6)$ & $0.051(5)$ & $0.443(16)$ \\
\hline $\mathrm{C} 46$ & $0.1644(17)$ & $0.0725(6)$ & $1.0117(5)$ & $0.072(4)$ & $0.443(16)$ \\
\hline $\mathrm{H} 46$ & 0.1145 & 0.0990 & 1.0077 & $0.086^{*}$ & $0.443(16)$ \\
\hline C47 & $0.1874(19)$ & $0.0520(7)$ & $1.0606(5)$ & $0.084(4)$ & $0.443(16)$ \\
\hline $\mathrm{H} 47$ & 0.1524 & 0.0646 & 1.0887 & $0.101 *$ & $0.443(16)$ \\
\hline $\mathrm{C} 48$ & $0.260(2)$ & $0.0147(8)$ & $1.0659(7)$ & $0.087(5)$ & $0.443(16)$ \\
\hline $\mathrm{H} 48$ & 0.2763 & 0.0016 & 1.0983 & $0.104 *$ & $0.443(16)$ \\
\hline $\mathrm{C} 49$ & $0.311(2)$ & $-0.0054(7)$ & $1.0249(6)$ & $0.087(5)$ & $0.443(16)$ \\
\hline $\mathrm{H} 49$ & 0.3585 & -0.0325 & 1.0289 & $0.105^{*}$ & $0.443(16)$ \\
\hline $\mathrm{C} 50$ & $0.288(2)$ & $0.0163(6)$ & $0.9768(6)$ & $0.074(4)$ & $0.443(16)$ \\
\hline
\end{tabular}




\begin{tabular}{|c|c|c|c|c|c|}
\hline $\mathrm{H} 50$ & 0.3257 & 0.0045 & 0.9489 & $0.088^{*}$ & $0.443(16)$ \\
\hline $\mathrm{C} 45 \mathrm{~B}$ & $0.2121(12)$ & $0.0535(6)$ & $0.9686(5)$ & $0.051(4)$ & $0.557(16)$ \\
\hline $\mathrm{C} 46 \mathrm{~B}$ & 0.1177 (13) & $0.0481(5)$ & $0.9986(5)$ & $0.078(4)$ & $0.557(16)$ \\
\hline H46B & 0.0400 & 0.0604 & 0.9879 & $0.094 *$ & $0.557(16)$ \\
\hline C47B & $0.1351(15)$ & $0.0244(6)$ & 1.0457 (6) & $0.093(4)$ & $0.557(16)$ \\
\hline H47B & 0.0696 & 0.0215 & 1.0661 & $0.111^{*}$ & $0.557(16)$ \\
\hline C48B & $0.2445(17)$ & $0.0062(8)$ & $1.0609(7)$ & $0.096(5)$ & $0.557(16)$ \\
\hline H48B & 0.2546 & -0.0107 & 1.0914 & $0.115^{*}$ & $0.557(16)$ \\
\hline C49B & $0.3440(13)$ & $0.0119(7)$ & $1.0321(6)$ & $0.092(4)$ & $0.557(16)$ \\
\hline H49B & 0.4226 & 0.0012 & 1.0440 & $0.111^{*}$ & $0.557(16)$ \\
\hline $\mathrm{C} 50 \mathrm{~B}$ & $0.3234(12)$ & $0.0342(6)$ & $0.9848(6)$ & $0.075(4)$ & $0.557(16)$ \\
\hline H50B & 0.3879 & 0.0359 & 0.9637 & $0.091 *$ & $0.557(16)$ \\
\hline P5 & $0.22108(12)$ & -0.05512 & $0.82795(5)$ & $0.0405(3)$ & \\
\hline C51 & 0.2753 (19) & $-0.0795(9)$ & $0.8929(8)$ & $0.057(4)$ & $0.443(16)$ \\
\hline C52 & 0.3948 (19) & $-0.0938(7)$ & $0.9079(8)$ & $0.062(4)$ & $0.443(16)$ \\
\hline H52 & 0.4552 & -0.0921 & 0.8848 & $0.075^{*}$ & $0.443(16)$ \\
\hline C53 & $0.4260(18)$ & -0.1108 (8) & $0.9572(8)$ & $0.071(5)$ & $0.443(16)$ \\
\hline $\mathrm{H} 53$ & 0.5068 & -0.1205 & 0.9670 & $0.085^{*}$ & $0.443(16)$ \\
\hline C54 & $0.3376(18)$ & $-0.1132(8)$ & $0.9911(7)$ & $0.080(4)$ & $0.443(16)$ \\
\hline H54 & 0.3568 & -0.1264 & 1.0233 & $0.095^{*}$ & $0.443(16)$ \\
\hline C55 & $0.2229(16)$ & $-0.0964(8)$ & $0.9781(6)$ & $0.081(4)$ & $0.443(16)$ \\
\hline H55 & 0.1663 & -0.0953 & 1.0028 & $0.098 *$ & $0.443(16)$ \\
\hline C56 & $0.1870(16)$ & $-0.0808(7)$ & $0.9287(6)$ & $0.070(4)$ & $0.443(16)$ \\
\hline H56 & 0.1057 & -0.0714 & 0.9196 & $0.085^{*}$ & $0.443(16)$ \\
\hline $\mathrm{C} 51 \mathrm{~B}$ & $0.2798(15)$ & $-0.0827(6)$ & $0.8872(6)$ & $0.052(3)$ & $0.557(16)$ \\
\hline $\mathrm{C} 52 \mathrm{~B}$ & $0.4062(15)$ & $-0.0814(5)$ & $0.9008(6)$ & $0.057(3)$ & $0.557(16)$ \\
\hline H52B & 0.4589 & -0.0671 & 0.8793 & $0.068 *$ & $0.557(16)$ \\
\hline C53B & $0.4541(16)$ & $-0.1015(6)$ & $0.9469(6)$ & 0.073 (4) & $0.557(16)$ \\
\hline H53B & 0.5390 & -0.1009 & 0.9566 & $0.087 *$ & $0.557(16)$ \\
\hline C54B & $0.3749(18)$ & $-0.1222(6)$ & $0.9777(6)$ & $0.083(4)$ & $0.557(16)$ \\
\hline H54B & 0.4074 & -0.1358 & 1.0084 & $0.099 *$ & $0.557(16)$ \\
\hline C55B & $0.2512(16)$ & $-0.1236(6)$ & $0.9653(5)$ & $0.090(4)$ & $0.557(16)$ \\
\hline H55B & 0.1997 & -0.1372 & 0.9877 & $0.108^{*}$ & $0.557(16)$ \\
\hline $\mathrm{C} 56 \mathrm{~B}$ & $0.2001(14)$ & $-0.1046(6)$ & $0.9187(5)$ & $0.075(3)$ & $0.557(16)$ \\
\hline H56B & 0.1153 & -0.1065 & 0.9091 & $0.089 *$ & $0.557(16)$ \\
\hline C57 & $0.0702(5)$ & $-0.0826(2)$ & $0.8134(2)$ & $0.0506(12)$ & \\
\hline C58 & $-0.0331(6)$ & -0.0548 & $0.8076(3)$ & 0.0708 (17) & \\
\hline H58 & -0.0237 & -0.0225 & 0.8083 & $0.085^{*}$ & \\
\hline C59 & $-0.1514(8)$ & $-0.0737(4)$ & 0.8007 (4) & $0.104(3)$ & \\
\hline H59 & -0.2208 & -0.0544 & 0.7965 & $0.124 *$ & \\
\hline C60 & $-0.1640(8)$ & $-0.1210(4)$ & $0.8003(4)$ & 0.109 (3) & \\
\hline H60 & -0.2429 & -0.1342 & 0.7981 & $0.131 *$ & \\
\hline C61 & $-0.0614(9)$ & $-0.1494(3)$ & $0.8031(4)$ & $0.113(3)$ & \\
\hline H61 & -0.0710 & -0.1817 & 0.8006 & $0.136^{*}$ & \\
\hline C62 & $0.0550(8)$ & $-0.1303(3)$ & $0.8094(4)$ & $0.093(3)$ & \\
\hline H62 & 0.1243 & -0.1497 & 0.8110 & $0.112^{*}$ & \\
\hline C63 & $0.3240(5)$ & $-0.08141(16)$ & $0.7838(2)$ & $0.0453(11)$ & \\
\hline H63A & 0.4096 & -0.0771 & 0.7975 & $0.054 *$ & \\
\hline
\end{tabular}




$\begin{array}{lllll}\text { H63B } & 0.3081 & -0.1148 & 0.7814 & 0.054^{*} \\ \text { P6 } & 0.30205(11) & -0.05569(4) & 0.71930(5) & 0.0373(2) \\ \text { C64 } & 0.1661(5) & -0.08506(17) & 0.6882(2) & 0.0430(11) \\ \text { C65 } & 0.0617(5) & -0.05952(18) & 0.6728(2) & 0.0525(13) \\ \text { H65 } & 0.0593 & -0.0276 & 0.6796 & 0.063^{*} \\ \text { C66 } & -0.0401(6) & -0.0814(2) & 0.6469(3) & 0.0690(18) \\ \text { H66 } & -0.1109 & -0.0641 & 0.6366 & 0.083^{*} \\ \text { C67 } & -0.0373(6) & -0.1274(2) & 0.6367(3) & 0.074(2) \\ \text { H67 } & -0.1056 & -0.1416 & 0.6188 & 0.089^{*} \\ \text { C68 } & 0.0650(6) & -0.1537(2) & 0.6523(3) & 0.0719(19) \\ \text { H68 } & 0.0653 & -0.1857 & 0.6458 & 0.086^{*} \\ \text { C69 } & 0.1676(5) & -0.13247(19) & 0.6776(3) & 0.0581(14) \\ \text { H69 } & 0.2380 & -0.1501 & 0.6875 & 0.070^{*} \\ \text { C70 } & 0.4268(5) & -0.07933(16) & 0.6847(2) & 0.0425(11) \\ \text { C71 } & 0.5398(5) & -0.0934(2) & 0.7075(3) & 0.0614(15) \\ \text { H71 } & 0.5536 & -0.0943 & 0.7431 & 0.074^{*} \\ \text { C72 } & 0.6333(6) & -0.1064(3) & 0.6777(3) & 0.0739(19) \\ \text { H72 } & 0.7096 & -0.1159 & 0.6936 & 0.089^{*} \\ \text { C73 } & 0.6153(6) & -0.1055(2) & 0.6256(3) & 0.0665(17) \\ \text { H73 } & 0.6790 & -0.1143 & 0.6060 & 0.080^{*} \\ \text { C74 } & 0.5047(6) & -0.0918(2) & 0.6024(2) & 0.0643(16) \\ \text { H74 } & 0.4919 & -0.0916 & 0.5667 & 0.077^{*} \\ \text { C75 } & 0.4095(6) & -0.0780(2) & 0.6315(2) & 0.0561(14) \\ \text { H75 } & 0.3342 & -0.0679 & 0.6152 & 0.067^{*} \\ \text { C76 } & 0.6973(9) & 0.2714(3) & 0.5417(3) & 0.088(2) \\ \text { C77 } & 0.8110(7) & 0.1979(3) & 0.5218(3) & 0.0730(17) \\ \text { C78 } & 0.7947(8) & 0.1618(3) & 0.4878(3) & 0.0845(19) \\ \text { H78 } & 0.7180 & 0.1578 & 0.4692 & 0.101^{*} \\ \text { C79 } & 0.8880(10) & 0.1320(4) & 0.4806(4) & 0.102(2) \\ \text { H79 } & 0.8755 & 0.1075 & 0.4572 & 0.122^{*} \\ \text { C80 } & 1.0011(10) & 0.1377(4) & 0.5078(4) & 0.105(2) \\ \text { H80 } & 1.0662 & 0.1174 & 0.5028 & 0.126^{*} \\ \text { C81 } & 1.0174(9) & 0.1724(4) & 0.5415(4) & 0.101(2) \\ \text { H81 } & 1.0945 & 0.1760 & 0.5599 & 0.121^{*} \\ \text { C82 } & 0.9232(7) & 0.2032(3) & 0.5500(3) & 0.0864(19) \\ \text { H82 } & 0.9356 & 0.2269 & 0.5743 & 0.104^{*} \\ & & & & \end{array}$

Atomic displacement parameters $\left(\AA^{2}\right)$

\begin{tabular}{lllllll}
\hline & $U^{11}$ & $U^{22}$ & $U^{33}$ & $U^{12}$ & $U^{13}$ & $U^{23}$ \\
\hline I1 & $0.03441(16)$ & $0.04363(16)$ & $0.04778(17)$ & $0.00246(12)$ & $-0.00487(12)$ & $-0.00638(12)$ \\
I2 & $0.03447(16)$ & $0.04649(17)$ & $0.05071(18)$ & $-0.00075(12)$ & $-0.00470(12)$ & $0.00563(13)$ \\
I3 & $0.0694(3)$ & $0.1116(4)$ & $0.0482(2)$ & $0.0199(2)$ & $0.00787(18)$ & $0.0156(2)$ \\
Ag1 & $0.0532(2)$ & $0.02985(16)$ & $0.04293(19)$ & $-0.00234(14)$ & $0.00843(15)$ & $0.00112(13)$ \\
Ag2 & $0.0511(2)$ & $0.03475(17)$ & $0.03267(17)$ & $-0.00435(14)$ & $0.00573(13)$ & $-0.00388(12)$ \\
Ag3 & $0.0500(2)$ & $0.03683(18)$ & $0.0495(2)$ & $0.00036(14)$ & $0.00783(15)$ & $-0.00519(14)$ \\
S1 & $0.148(2)$ & $0.0834(14)$ & $0.1021(16)$ & $-0.0216(14)$ & $0.0263(15)$ & $0.0202(12)$ \\
N1 & $0.115(6)$ & $0.109(6)$ & $0.097(5)$ & $0.036(5)$ & $0.029(4)$ & $0.034(4)$
\end{tabular}




\begin{tabular}{|c|c|c|c|c|c|c|}
\hline N2 & $0.078(4)$ & $0.081(4)$ & $0.075(4)$ & $0.000(3)$ & $0.006(3)$ & $0.010(3)$ \\
\hline P1 & $0.0388(6)$ & $0.0306(5)$ & $0.0325(5)$ & $-0.0033(4)$ & $0.0029(4)$ & $-0.0020(4)$ \\
\hline $\mathrm{C} 1$ & $0.046(3)$ & $0.036(2)$ & $0.041(3)$ & $-0.003(2)$ & $0.001(2)$ & $-0.0086(19)$ \\
\hline $\mathrm{C} 2$ & $0.053(4)$ & $0.099(5)$ & $0.050(3)$ & $-0.013(3)$ & $-0.003(3)$ & $-0.016(3)$ \\
\hline $\mathrm{C} 3$ & $0.063(4)$ & $0.125(7)$ & $0.073(5)$ & $-0.025(4)$ & -0.012 & $-0.023(4)$ \\
\hline $\mathrm{C} 4$ & $0.083(5)$ & $0.107(6)$ & $0.065(4)$ & $-0.013(4)$ & $-0.012(4)$ & $-0.037(4)$ \\
\hline $\mathrm{C} 5$ & $0.093(6)$ & $0.140(8)$ & $0.065(5)$ & $-0.015(6)$ & 0.009 (4) & $-0.046(5)$ \\
\hline C6 & $0.071(4)$ & $0.118(6)$ & $0.052(4)$ & $-0.035(4)$ & $0.015(3)$ & $-0.036(4)$ \\
\hline $\mathrm{C} 7$ & $0.044(3)$ & $0.035(2)$ & $0.033(2)$ & -0.0017 (19) & $0.0034(18)$ & $-0.0028(18)$ \\
\hline $\mathrm{C} 8$ & $0.049(3)$ & $0.048(3)$ & $0.058(3)$ & $0.001(2)$ & $0.010(2)$ & $0.005(2)$ \\
\hline C9 & $0.059(4)$ & $0.071(4)$ & $0.065(4)$ & $0.015(3)$ & $-0.003(3)$ & $0.002(3)$ \\
\hline C10 & $0.039(3)$ & $0.089(5)$ & $0.064(4)$ & $0.005(3)$ & $0.006(3)$ & $-0.014(3)$ \\
\hline C11 & $0.047(3)$ & $0.081(4)$ & $0.050(3)$ & $-0.014(3)$ & $0.010(2)$ & $0.000(3)$ \\
\hline $\mathrm{C} 12$ & $0.048(3)$ & $0.055(3)$ & $0.042(3)$ & $-0.005(2)$ & $0.006(2)$ & $0.003(2)$ \\
\hline C13 & $0.046(3)$ & $0.032(2)$ & $0.031(2)$ & $-0.0008(19)$ & $-0.0017(18)$ & $0.0014(17)$ \\
\hline $\mathrm{P} 2$ & $0.0429(6)$ & $0.0291(5)$ & $0.0302(5)$ & -0.0023 & $0.0024(4)$ & $-0.0003(4)$ \\
\hline C14 & $0.050(3)$ & $0.035(2)$ & $0.035(2)$ & $-0.008(2)$ & $0.001(2)$ & $-0.0046(18)$ \\
\hline $\mathrm{C} 15$ & $0.049(3)$ & $0.051(3)$ & $0.043(3)$ & $-0.006(2)$ & $0.005(2)$ & $0.001(2)$ \\
\hline $\mathrm{C} 16$ & $0.050(3)$ & $0.079(4)$ & $0.057(3)$ & $-0.001(3)$ & $-0.007(3)$ & -0.005 \\
\hline $\mathrm{C} 17$ & $0.053(4)$ & $0.105(5)$ & $0.054(3)$ & -0.030 & $0.007(3)$ & $-0.011(3)$ \\
\hline $\mathrm{C} 18$ & $0.074(4)$ & $0.088(5)$ & $0.059(4)$ & -0.035 & $0.011(3)$ & $0.011(3)$ \\
\hline C19 & $0.065(4)$ & $0.057(3)$ & $0.046(3)$ & -0.015 & $-0.002(2)$ & $0.012(2)$ \\
\hline $\mathrm{C} 20$ & 0.055 & $0.029(2)$ & $0.043(3)$ & $0.001(2)$ & $0.000(2)$ & 0.0002 (19) \\
\hline $\mathrm{C} 21$ & $0.092(5)$ & $0.047(3)$ & $0.049(3)$ & $0.013(3)$ & $-0.002(3)$ & $-0.003(2)$ \\
\hline $\mathrm{C} 22$ & $0.111(6)$ & $0.052(3)$ & $0.063(4)$ & $0.017(4)$ & $-0.019(4)$ & $0.009(3)$ \\
\hline $\mathrm{C} 23$ & $0.080(5)$ & $0.038(3)$ & $0.112(6)$ & $0.015(3)$ & $-0.010(4)$ & $-0.001(3)$ \\
\hline $\mathrm{C} 24$ & $0.089(5)$ & $0.052(4)$ & $0.086(5)$ & $0.020(3)$ & $0.025(4)$ & $0.002(3)$ \\
\hline $\mathrm{C} 25$ & $0.083(4)$ & $0.038(3)$ & $0.060(3)$ & $0.010(3)$ & $0.016(3)$ & $0.002(2)$ \\
\hline P3 & $0.0323(6)$ & $0.0296(5)$ & $0.0298(5)$ & -0.0010 & $0.0018(4)$ & $-0.0039(4)$ \\
\hline $\mathrm{C} 26$ & $0.032(2)$ & $0.028(2)$ & $0.040(2)$ & $0.0036(17)$ & $-0.0023(18)$ & $-0.0028(17)$ \\
\hline $\mathrm{C} 27$ & $0.042(3)$ & $0.039(2)$ & $0.043(3)$ & $-0.002(2)$ & $0.002(2)$ & $-0.005(2)$ \\
\hline $\mathrm{C} 28$ & $0.041(3)$ & $0.049(3)$ & $0.068(4)$ & $-0.006(2)$ & $0.011(2)$ & $0.003(3)$ \\
\hline $\mathrm{C} 29$ & $0.034(3)$ & $0.042(3)$ & $0.081(4)$ & $-0.003(2)$ & $-0.003(3)$ & -0.006 \\
\hline $\mathrm{C} 30$ & $0.046(3)$ & $0.051(3)$ & $0.056(3)$ & $0.001(2)$ & $-0.013(2)$ & $-0.014(2)$ \\
\hline $\mathrm{C} 31$ & $0.046(3)$ & $0.045(3)$ & $0.044(3)$ & $-0.006(2)$ & $0.001(2)$ & $-0.009(2)$ \\
\hline $\mathrm{C} 32$ & $0.034(2)$ & $0.032(2)$ & $0.033(2)$ & $0.0006(17)$ & $0.0051(17)$ & $-0.0008(17)$ \\
\hline $\mathrm{C} 33$ & $0.050(3)$ & $0.047(3)$ & $0.062(3)$ & $0.005(2)$ & $-0.003(2)$ & $-0.018(2)$ \\
\hline C34 & $0.057(4)$ & $0.050(3)$ & $0.090(5)$ & 0.010 & $0.004(3)$ & -0.026 \\
\hline C35 & 0.048 & $0.053(3)$ & $0.075(4)$ & $0.017(3)$ & $0.006(3)$ & -0.001 \\
\hline $\mathrm{C} 36$ & $0.041(3)$ & $0.065(3)$ & $0.057(3)$ & $0.007(2)$ & $-0.003(2)$ & -0.003 \\
\hline $\mathrm{C} 37$ & $0.049(3)$ & $0.043(3)$ & $0.042(3)$ & $0.002(2)$ & $0.000(2)$ & $-0.013(2)$ \\
\hline C38 & $0.038(2)$ & $0.037(2)$ & $0.034(2)$ & $-0.0023(19)$ & $0.0022(18)$ & $-0.0020(18)$ \\
\hline P4 & $0.0374(6)$ & $0.0321(5)$ & $0.0352(6)$ & -0.0001 & 0.0037 (4) & $0.0004(4)$ \\
\hline C39 & $0.038(2)$ & $0.038(2)$ & $0.039(2)$ & $0.0001(19)$ & 0.0077 (19) & 0.0065 (19) \\
\hline $\mathrm{C} 40$ & $0.053(3)$ & $0.047(3)$ & $0.048(3)$ & $0.002(2)$ & $0.014(2)$ & $0.001(2)$ \\
\hline C41 & $0.056(4)$ & $0.053(3)$ & $0.071(4)$ & $0.015(3)$ & $0.023(3)$ & $0.010(3)$ \\
\hline $\mathrm{C} 42$ & $0.044(3)$ & $0.069(4)$ & $0.081(4)$ & $0.017(3)$ & $0.012(3)$ & $0.023(3)$ \\
\hline $\mathrm{C} 43$ & $0.036(3)$ & $0.073(4)$ & $0.082(4)$ & $-0.007(3)$ & -0.010 & $0.013(3)$ \\
\hline
\end{tabular}




\begin{tabular}{|c|c|c|c|c|c|c|}
\hline $\mathrm{C} 44$ & $0.047(3)$ & $0.048(3)$ & $0.061(3)$ & $-0.004(2)$ & $0.002(2)$ & $0.000(2)$ \\
\hline $\mathrm{C} 45$ & $0.086(9)$ & $0.040(7)$ & $0.026(6)$ & $0.011(7)$ & $-0.002(7)$ & $0.001(6)$ \\
\hline $\mathrm{C} 46$ & $0.104(9)$ & $0.072(7)$ & $0.040(6)$ & $0.018(7)$ & $0.010(6)$ & $0.012(5)$ \\
\hline $\mathrm{C} 47$ & $0.117(9)$ & $0.089(8)$ & $0.045(6)$ & $0.012(7)$ & $0.007(6)$ & $0.019(6)$ \\
\hline C48 & $0.123(10)$ & $0.084(8)$ & $0.048(7)$ & $0.009(8)$ & $-0.017(7)$ & $0.019(6)$ \\
\hline $\mathrm{C} 49$ & $0.122(10)$ & $0.073(8)$ & $0.061(7)$ & $0.020(8)$ & $-0.022(7)$ & $0.009(6)$ \\
\hline $\mathrm{C} 50$ & $0.110(10)$ & $0.062(7)$ & $0.046(6)$ & $0.017(7)$ & $-0.006(7)$ & $0.000(6)$ \\
\hline C45B & $0.037(5)$ & $0.054(7)$ & $0.061(7)$ & $-0.007(5)$ & $0.004(5)$ & $0.023(6)$ \\
\hline C46B & $0.076(6)$ & 0.088 (7) & $0.073(7)$ & $0.006(6)$ & $0.014(5)$ & $0.035(6)$ \\
\hline C47B & $0.100(7)$ & $0.107(8)$ & $0.076(7)$ & $-0.001(7)$ & $0.027(6)$ & $0.043(6)$ \\
\hline C48B & $0.100(8)$ & $0.103(8)$ & $0.084(8)$ & $-0.009(7)$ & $0.001(7)$ & $0.050(7)$ \\
\hline C49B & $0.075(7)$ & $0.100(8)$ & $0.097(8)$ & $-0.008(6)$ & $-0.019(6)$ & $0.042(7)$ \\
\hline $\mathrm{C} 50 \mathrm{~B}$ & $0.054(6)$ & $0.083(7)$ & $0.087(7)$ & $-0.001(5)$ & $-0.005(5)$ & $0.041(6)$ \\
\hline P5 & $0.0442(7)$ & $0.0332(6)$ & $0.0442(6)$ & $-0.0012(5)$ & $0.0048(5)$ & $0.0049(5)$ \\
\hline $\mathrm{C} 51$ & $0.065(8)$ & $0.056(8)$ & $0.049(8)$ & $0.010(7)$ & $0.007(7)$ & $0.016(6)$ \\
\hline $\mathrm{C} 52$ & $0.070(8)$ & $0.067(9)$ & $0.051(8)$ & $0.013(6)$ & $0.012(7)$ & $0.009(6)$ \\
\hline C53 & $0.079(8)$ & $0.082(9)$ & $0.054(8)$ & $0.014(7)$ & $0.013(7)$ & $0.014(7)$ \\
\hline $\mathrm{C} 54$ & $0.087(8)$ & $0.095(9)$ & $0.057(8)$ & $0.015(7)$ & $0.012(7)$ & $0.020(7)$ \\
\hline $\mathrm{C} 55$ & $0.089(8)$ & $0.094(9)$ & $0.063(7)$ & $0.010(7)$ & $0.018(6)$ & $0.025(7)$ \\
\hline $\mathrm{C} 56$ & $0.079(8)$ & $0.075(8)$ & $0.059(7)$ & $0.005(6)$ & $0.015(6)$ & $0.029(6)$ \\
\hline C51B & $0.068(7)$ & $0.045(6)$ & $0.042(6)$ & $-0.006(5)$ & $-0.002(5)$ & $0.005(5)$ \\
\hline C52B & $0.074(7)$ & $0.045(6)$ & $0.049(6)$ & $0.007(5)$ & $-0.009(5)$ & $0.009(5)$ \\
\hline C53B & $0.094(8)$ & $0.068(6)$ & $0.053(7)$ & $0.013(6)$ & $-0.011(6)$ & $0.014(5)$ \\
\hline C54B & $0.109(8)$ & $0.083(7)$ & $0.054(7)$ & $0.012(6)$ & $-0.009(7)$ & $0.022(6)$ \\
\hline C55B & $0.117(8)$ & $0.089(7)$ & $0.062(6)$ & $-0.006(7)$ & $0.005(6)$ & $0.027(6)$ \\
\hline C56B & $0.091(7)$ & $0.075(7)$ & $0.058(6)$ & $-0.009(6)$ & $0.007(5)$ & $0.019(5)$ \\
\hline $\mathrm{C} 57$ & $0.051(3)$ & $0.055(3)$ & $0.047(3)$ & -0.009 (2) & $0.005(2)$ & 0.007 (2) \\
\hline $\mathrm{C} 58$ & $0.056(4)$ & $0.076(4)$ & $0.080(4)$ & $-0.005(3)$ & $0.003(3)$ & $-0.010(3)$ \\
\hline $\mathrm{C} 59$ & $0.057(5)$ & $0.125(8)$ & $0.127(8)$ & $-0.004(5)$ & $-0.003(4)$ & $-0.010(6)$ \\
\hline $\mathrm{C} 60$ & $0.064(5)$ & $0.144(9)$ & $0.116(7)$ & $-0.049(6)$ & $-0.003(5)$ & $0.014(6)$ \\
\hline C61 & $0.099(7)$ & $0.082(6)$ & $0.154(9)$ & $-0.047(5)$ & $-0.021(6)$ & $0.023(6)$ \\
\hline C62 & $0.079(5)$ & $0.053(4)$ & $0.143(8)$ & $-0.022(4)$ & $-0.013(5)$ & $0.029(4)$ \\
\hline C63 & $0.053(3)$ & $0.031(2)$ & $0.053(3)$ & $0.000(2)$ & $0.005(2)$ & $0.002(2)$ \\
\hline P6 & $0.0394(6)$ & $0.0265(5)$ & $0.0462(6)$ & $-0.0036(4)$ & $0.0056(5)$ & $-0.0022(4)$ \\
\hline C64 & $0.043(3)$ & $0.036(2)$ & $0.051(3)$ & $-0.006(2)$ & $0.007(2)$ & $-0.004(2)$ \\
\hline $\mathrm{C} 65$ & $0.046(3)$ & $0.037(3)$ & $0.075(4)$ & $-0.004(2)$ & 0.009 (3) & $-0.014(2)$ \\
\hline C66 & $0.044(3)$ & $0.059(4)$ & $0.103(5)$ & $0.000(3)$ & $-0.003(3)$ & $-0.014(3)$ \\
\hline C67 & $0.046(3)$ & $0.062(4)$ & $0.115(6)$ & $-0.012(3)$ & $0.004(3)$ & -0.030 \\
\hline C68 & $0.056(4)$ & $0.047(3)$ & $0.114(6)$ & $-0.017(3)$ & $0.013(3)$ & $-0.030(3)$ \\
\hline C69 & $0.049(3)$ & $0.036(3)$ & $0.089(4)$ & $-0.005(2)$ & $0.005(3)$ & $-0.005(3)$ \\
\hline $\mathrm{C} 70$ & $0.045(3)$ & $0.030(2)$ & $0.053(3)$ & $-0.0053(19)$ & $0.009(2)$ & $-0.006(2)$ \\
\hline C71 & $0.042(3)$ & $0.076(4)$ & $0.066(4)$ & $0.004(3)$ & $0.006(3)$ & $-0.007(3)$ \\
\hline C72 & $0.046(4)$ & $0.090(5)$ & $0.087(5)$ & $0.006(3)$ & 0.009 (3) & $-0.007(4)$ \\
\hline $\mathrm{C} 73$ & $0.054(4)$ & $0.074(4)$ & $0.075(4)$ & $-0.005(3)$ & $0.025(3)$ & $-0.021(3)$ \\
\hline C74 & $0.067(4)$ & $0.071(4)$ & $0.057(3)$ & $-0.011(3)$ & $0.018(3)$ & $-0.014(3)$ \\
\hline $\mathrm{C} 75$ & $0.051(3)$ & $0.058(3)$ & $0.061(3)$ & $0.001(3)$ & $0.008(3)$ & $-0.004(3)$ \\
\hline C76 & 0.109 (6) & $0.096(6)$ & $0.061(4)$ & $0.014(5)$ & $0.019(4)$ & $0.030(4)$ \\
\hline C77 & 0.073 & $0.081(4)$ & $0.066(4)$ & $0.001(3)$ & $0.011(3)$ & 0.025 \\
\hline
\end{tabular}




\begin{tabular}{lllllll} 
C78 & $0.094(5)$ & $0.092(5)$ & $0.067(4)$ & $0.012(4)$ & $0.002(3)$ & $0.013(3)$ \\
C79 & $0.113(5)$ & $0.102(5)$ & $0.092(5)$ & $0.009(5)$ & $0.014(4)$ & $0.011(4)$ \\
C80 & $0.098(5)$ & $0.108(5)$ & $0.112(6)$ & $0.012(5)$ & $0.029(4)$ & $0.020(5)$ \\
C81 & $0.077(4)$ & $0.113(5)$ & $0.113(5)$ & $-0.002(4)$ & $0.007(4)$ & $0.016(5)$ \\
C82 & $0.073(4)$ & $0.095(5)$ & $0.090(4)$ & $-0.005(4)$ & $0.004(4)$ & $0.013(4)$ \\
\hline
\end{tabular}

Geometric parameters $\left(\hat{A},{ }^{\circ}\right)$

\begin{tabular}{|c|c|c|c|}
\hline $\mathrm{I} 1-\mathrm{Ag} 1$ & $2.8975(5)$ & $\mathrm{C} 40-\mathrm{C} 41$ & $1.397(8)$ \\
\hline $\mathrm{I} 1-\mathrm{Ag} 2$ & $2.9687(5)$ & $\mathrm{C} 40-\mathrm{H} 40$ & 0.9300 \\
\hline $\mathrm{I} 1-\mathrm{Ag} 3$ & $2.9916(5)$ & $\mathrm{C} 41-\mathrm{C} 42$ & $1.356(9)$ \\
\hline $\mathrm{I} 2-\mathrm{Ag} 3$ & $2.9686(5)$ & $\mathrm{C} 41-\mathrm{H} 41$ & 0.9300 \\
\hline $\mathrm{I} 2-\mathrm{Ag} 1$ & $2.9711(5)$ & $\mathrm{C} 42-\mathrm{C} 43$ & $1.378(10)$ \\
\hline $\mathrm{I} 2-\mathrm{Ag} 2$ & $3.0455(5)$ & $\mathrm{C} 42-\mathrm{H} 42$ & 0.9300 \\
\hline $\mathrm{Ag} 1-\mathrm{P} 6$ & $2.4614(11)$ & $\mathrm{C} 43-\mathrm{C} 44$ & $1.388(8)$ \\
\hline $\mathrm{Ag} 1-\mathrm{P} 1$ & $2.4621(12)$ & $\mathrm{C} 43-\mathrm{H} 43$ & 0.9300 \\
\hline $\mathrm{Ag} 1-\mathrm{Ag} 2$ & $3.0823(5)$ & $\mathrm{C} 44-\mathrm{H} 44$ & 0.9300 \\
\hline $\mathrm{Ag} 1-\mathrm{Ag} 3$ & $3.2026(5)$ & $\mathrm{C} 45-\mathrm{C} 50$ & $1.350(15)$ \\
\hline $\mathrm{Ag} 2-\mathrm{P} 3$ & $2.4690(11)$ & $\mathrm{C} 45-\mathrm{C} 46$ & $1.357(15)$ \\
\hline $\mathrm{Ag} 2-\mathrm{P} 2$ & $2.4836(11)$ & $\mathrm{C} 46-\mathrm{C} 47$ & $1.408(15)$ \\
\hline $\mathrm{Ag} 2-\mathrm{Ag} 3$ & $3.2999(5)$ & $\mathrm{C} 46-\mathrm{H} 46$ & 0.9300 \\
\hline $\mathrm{Ag} 3-\mathrm{P} 4$ & $2.4679(12)$ & $\mathrm{C} 47-\mathrm{C} 48$ & $1.321(18)$ \\
\hline Ag3-P5 & $2.4753(12)$ & $\mathrm{C} 47-\mathrm{H} 47$ & 0.9300 \\
\hline $\mathrm{S} 1-\mathrm{C} 76$ & $1.651(10)$ & $\mathrm{C} 48-\mathrm{C} 49$ & $1.378(19)$ \\
\hline $\mathrm{N} 1-\mathrm{C} 76$ & $1.355(11)$ & $\mathrm{C} 48-\mathrm{H} 48$ & 0.9300 \\
\hline $\mathrm{N} 1-\mathrm{H} 1 \mathrm{~A}$ & 0.8600 & $\mathrm{C} 49-\mathrm{C} 50$ & $1.404(15)$ \\
\hline N1-H1B & 0.8600 & $\mathrm{C} 49-\mathrm{H} 49$ & 0.9300 \\
\hline $\mathrm{N} 2-\mathrm{C} 76$ & $1.343(11)$ & $\mathrm{C} 50-\mathrm{H} 50$ & 0.9300 \\
\hline $\mathrm{N} 2-\mathrm{C} 77$ & $1.432(10)$ & $\mathrm{C} 45 \mathrm{~B}-\mathrm{C} 46 \mathrm{~B}$ & $1.354(14)$ \\
\hline $\mathrm{N} 2-\mathrm{H} 2 \mathrm{~A}$ & 0.8600 & $\mathrm{C} 45 \mathrm{~B}-\mathrm{C} 50 \mathrm{~B}$ & $1.356(13)$ \\
\hline $\mathrm{P} 1-\mathrm{C} 7$ & $1.814(5)$ & $\mathrm{C} 46 \mathrm{~B}-\mathrm{C} 47 \mathrm{~B}$ & $1.403(14)$ \\
\hline $\mathrm{P} 1-\mathrm{C} 1$ & $1.836(5)$ & $\mathrm{C} 46 \mathrm{~B}-\mathrm{H} 46 \mathrm{~B}$ & 0.9300 \\
\hline $\mathrm{P} 1-\mathrm{C} 13$ & $1.838(4)$ & $\mathrm{C} 47 \mathrm{~B}-\mathrm{C} 48 \mathrm{~B}$ & $1.320(17)$ \\
\hline $\mathrm{C} 1-\mathrm{C} 6$ & $1.359(8)$ & $\mathrm{C} 47 \mathrm{~B}-\mathrm{H} 47 \mathrm{~B}$ & 0.9300 \\
\hline $\mathrm{C} 1-\mathrm{C} 2$ & $1.373(8)$ & $\mathrm{C} 48 \mathrm{~B}-\mathrm{C} 49 \mathrm{~B}$ & $1.379(18)$ \\
\hline $\mathrm{C} 2-\mathrm{C} 3$ & $1.380(9)$ & $\mathrm{C} 48 \mathrm{~B}-\mathrm{H} 48 \mathrm{~B}$ & 0.9300 \\
\hline $\mathrm{C} 2-\mathrm{H} 2$ & 0.9300 & $\mathrm{C} 49 \mathrm{~B}-\mathrm{C} 50 \mathrm{~B}$ & $1.393(14)$ \\
\hline $\mathrm{C} 3-\mathrm{C} 4$ & $1.368(11)$ & $\mathrm{C} 49 \mathrm{~B}-\mathrm{H} 49 \mathrm{~B}$ & 0.9300 \\
\hline $\mathrm{C} 3-\mathrm{H} 3$ & 0.9300 & $\mathrm{C} 50 \mathrm{~B}-\mathrm{H} 50 \mathrm{~B}$ & 0.9300 \\
\hline $\mathrm{C} 4-\mathrm{C} 5$ & $1.324(11)$ & $\mathrm{P} 5-\mathrm{C} 51 \mathrm{~B}$ & $1.803(15)$ \\
\hline $\mathrm{C} 4-\mathrm{H} 4$ & 0.9300 & $\mathrm{P} 5-\mathrm{C} 57$ & $1.819(6)$ \\
\hline $\mathrm{C} 5-\mathrm{C} 6$ & $1.388(9)$ & $\mathrm{P} 5-\mathrm{C} 63$ & $1.838(5)$ \\
\hline $\mathrm{C} 5-\mathrm{H} 5$ & 0.9300 & $\mathrm{P} 5-\mathrm{C} 51$ & $1.879(19)$ \\
\hline C6- 6 6 & 0.9300 & $\mathrm{C} 51-\mathrm{C} 52$ & $1.378(15)$ \\
\hline $\mathrm{C} 7-\mathrm{C} 8$ & $1.386(7)$ & $\mathrm{C} 51-\mathrm{C} 56$ & $1.400(16)$ \\
\hline $\mathrm{C} 7-\mathrm{C} 12$ & $1.395(7)$ & $\mathrm{C} 52-\mathrm{C} 53$ & $1.390(16)$ \\
\hline $\mathrm{C} 8-\mathrm{C} 9$ & $1.385(9)$ & $\mathrm{C} 52-\mathrm{H} 52$ & 0.9300 \\
\hline $\mathrm{C} 8-\mathrm{H} 8$ & 0.9300 & $\mathrm{C} 53-\mathrm{C} 54$ & $1.365(19)$ \\
\hline
\end{tabular}




\begin{tabular}{|c|c|c|c|}
\hline $\mathrm{C} 9-\mathrm{C} 10$ & $1.363(10)$ & $\mathrm{C} 53-\mathrm{H} 53$ & 0.9300 \\
\hline C9-H9 & 0.9300 & $\mathrm{C} 54-\mathrm{C} 55$ & $1.343(19)$ \\
\hline $\mathrm{C} 10-\mathrm{C} 11$ & $1.366(9)$ & $\mathrm{C} 54-\mathrm{H} 54$ & 0.9300 \\
\hline $\mathrm{C} 10-\mathrm{H} 10$ & 0.9300 & $\mathrm{C} 55-\mathrm{C} 56$ & $1.389(15)$ \\
\hline $\mathrm{C} 11-\mathrm{C} 12$ & $1.382(8)$ & $\mathrm{C} 55-\mathrm{H} 55$ & 0.9300 \\
\hline $\mathrm{C} 11-\mathrm{H} 11$ & 0.9300 & C56-H56 & 0.9300 \\
\hline $\mathrm{C} 12-\mathrm{H} 12$ & 0.9300 & $\mathrm{C} 51 \mathrm{~B}-\mathrm{C} 52 \mathrm{~B}$ & $1.381(13)$ \\
\hline $\mathrm{C} 13-\mathrm{P} 2$ & $1.838(4)$ & $\mathrm{C} 51 \mathrm{~B}-\mathrm{C} 56 \mathrm{~B}$ & $1.394(14)$ \\
\hline $\mathrm{C} 13-\mathrm{H} 13 \mathrm{~A}$ & 0.9700 & $\mathrm{C} 52 \mathrm{~B}-\mathrm{C} 53 \mathrm{~B}$ & $1.393(13)$ \\
\hline C13-H13B & 0.9700 & $\mathrm{C} 52 \mathrm{~B}-\mathrm{H} 52 \mathrm{~B}$ & 0.9300 \\
\hline $\mathrm{P} 2-\mathrm{C} 14$ & $1.824(5)$ & $\mathrm{C} 53 \mathrm{~B}-\mathrm{C} 54 \mathrm{~B}$ & $1.363(18)$ \\
\hline $\mathrm{P} 2-\mathrm{C} 20$ & $1.832(5)$ & $\mathrm{C} 53 \mathrm{~B}-\mathrm{H} 53 \mathrm{~B}$ & 0.9300 \\
\hline $\mathrm{C} 14-\mathrm{C} 19$ & $1.387(7)$ & $\mathrm{C} 54 \mathrm{~B}-\mathrm{C} 55 \mathrm{~B}$ & 1.349 (19) \\
\hline $\mathrm{C} 14-\mathrm{C} 15$ & $1.393(7)$ & $\mathrm{C} 54 \mathrm{~B}-\mathrm{H} 54 \mathrm{~B}$ & 0.9300 \\
\hline $\mathrm{C} 15-\mathrm{C} 16$ & $1.380(8)$ & $\mathrm{C} 55 \mathrm{~B}-\mathrm{C} 56 \mathrm{~B}$ & $1.400(14)$ \\
\hline $\mathrm{C} 15-\mathrm{H} 15$ & 0.9300 & $\mathrm{C} 55 \mathrm{~B}-\mathrm{H} 55 \mathrm{~B}$ & 0.9300 \\
\hline $\mathrm{C} 16-\mathrm{C} 17$ & $1.371(10)$ & $\mathrm{C} 56 \mathrm{~B}-\mathrm{H} 56 \mathrm{~B}$ & 0.9300 \\
\hline $\mathrm{C} 16-\mathrm{H} 16$ & 0.9300 & $\mathrm{C} 57-\mathrm{C} 58$ & $1.366(9)$ \\
\hline $\mathrm{C} 17-\mathrm{C} 18$ & $1.373(10)$ & $\mathrm{C} 57-\mathrm{C} 62$ & $1.376(9)$ \\
\hline C17-H17 & 0.9300 & $\mathrm{C} 58-\mathrm{C} 59$ & $1.385(11)$ \\
\hline $\mathrm{C} 18-\mathrm{C} 19$ & $1.373(9)$ & $\mathrm{C} 58-\mathrm{H} 58$ & 0.9300 \\
\hline $\mathrm{C} 18-\mathrm{H} 18$ & 0.9300 & $\mathrm{C} 59-\mathrm{C} 60$ & $1.358(14)$ \\
\hline C19-H19 & 0.9300 & $\mathrm{C} 59-\mathrm{H} 59$ & 0.9300 \\
\hline $\mathrm{C} 20-\mathrm{C} 21$ & $1.380(8)$ & $\mathrm{C} 60-\mathrm{C} 61$ & $1.371(14)$ \\
\hline $\mathrm{C} 20-\mathrm{C} 25$ & $1.383(8)$ & $\mathrm{C} 60-\mathrm{H} 60$ & 0.9300 \\
\hline $\mathrm{C} 21-\mathrm{C} 22$ & $1.378(9)$ & C61-C62 & $1.368(11)$ \\
\hline $\mathrm{C} 21-\mathrm{H} 21$ & 0.9300 & C61-H61 & 0.9300 \\
\hline $\mathrm{C} 22-\mathrm{C} 23$ & $1.388(11)$ & $\mathrm{C} 62-\mathrm{H} 62$ & 0.9300 \\
\hline $\mathrm{C} 22-\mathrm{H} 22$ & 0.9300 & C63-P6 & $1.835(5)$ \\
\hline $\mathrm{C} 23-\mathrm{C} 24$ & $1.350(10)$ & $\mathrm{C} 63-\mathrm{H} 63 \mathrm{~A}$ & 0.9700 \\
\hline $\mathrm{C} 23-\mathrm{H} 23$ & 0.9300 & C63-H63B & 0.9700 \\
\hline $\mathrm{C} 24-\mathrm{C} 25$ & $1.403(9)$ & P6-C64 & $1.818(5)$ \\
\hline $\mathrm{C} 24-\mathrm{H} 24$ & 0.9300 & $\mathrm{P} 6-\mathrm{C} 70$ & $1.824(5)$ \\
\hline $\mathrm{C} 25-\mathrm{H} 25$ & 0.9300 & $\mathrm{C} 64-\mathrm{C} 65$ & $1.373(8)$ \\
\hline $\mathrm{P} 3-\mathrm{C} 26$ & $1.822(4)$ & C64-C69 & $1.383(7)$ \\
\hline P3-C32 & $1.837(4)$ & $\mathrm{C} 65-\mathrm{C} 66$ & $1.388(8)$ \\
\hline P3-C38 & $1.845(5)$ & C65-H65 & 0.9300 \\
\hline $\mathrm{C} 26-\mathrm{C} 27$ & $1.390(7)$ & C66-C67 & $1.342(9)$ \\
\hline $\mathrm{C} 26-\mathrm{C} 31$ & $1.400(6)$ & C66-H66 & 0.9300 \\
\hline $\mathrm{C} 27-\mathrm{C} 28$ & $1.385(7)$ & C67-C68 & $1.366(10)$ \\
\hline $\mathrm{C} 27-\mathrm{H} 27$ & 0.9300 & C67-H67 & 0.9300 \\
\hline $\mathrm{C} 28-\mathrm{C} 29$ & $1.363(8)$ & C68-C69 & $1.378(8)$ \\
\hline $\mathrm{C} 28-\mathrm{H} 28$ & 0.9300 & C68-H68 & 0.9300 \\
\hline $\mathrm{C} 29-\mathrm{C} 30$ & $1.377(9)$ & C69-H69 & 0.9300 \\
\hline $\mathrm{C} 29-\mathrm{H} 29$ & 0.9300 & $\mathrm{C} 70-\mathrm{C} 71$ & $1.370(8)$ \\
\hline $\mathrm{C} 30-\mathrm{C} 31$ & $1.386(7)$ & $\mathrm{C} 70-\mathrm{C} 75$ & $1.388(8)$ \\
\hline $\mathrm{C} 30-\mathrm{H} 30$ & 0.9300 & $\mathrm{C} 71-\mathrm{C} 72$ & $1.382(9)$ \\
\hline $\mathrm{C} 31-\mathrm{H} 31$ & 0.9300 & C71-H71 & 0.9300 \\
\hline
\end{tabular}




\section{C $32-\mathrm{C} 37$ \\ $\mathrm{C} 32-\mathrm{C} 33$ \\ $\mathrm{C} 33-\mathrm{C} 34$ \\ C $33-\mathrm{H} 33$ \\ C $34-\mathrm{C} 35$ \\ $\mathrm{C} 34-\mathrm{H} 34$ \\ C $35-\mathrm{C} 36$ \\ $\mathrm{C} 35-\mathrm{H} 35$ \\ C $36-\mathrm{C} 37$ \\ C36- 336 \\ C $37-\mathrm{H} 37$ \\ C38-P4 \\ $\mathrm{C} 38-\mathrm{H} 38 \mathrm{~A}$ \\ C38-H38B \\ P4-C45B \\ P4-C39 \\ $\mathrm{P} 4-\mathrm{C} 45$ \\ $\mathrm{C} 39-\mathrm{C} 44$ \\ $\mathrm{C} 39-\mathrm{C} 40$}

Ag1-I1-Ag2

Ag1-I1-Ag3

Ag2-I1-Ag3

Ag3-I2-Ag1

$\mathrm{Ag} 3-\mathrm{I} 2-\mathrm{Ag} 2$

$\mathrm{Ag} 1-\mathrm{I} 2-\mathrm{Ag} 2$

P6-Ag1-P1

P6-Ag1-I1

P1-Ag1-I1

P6-Ag1-I2

P1-Ag1-I2

I1-Ag1-I2

P6-Ag1-Ag2

$\mathrm{P} 1-\mathrm{Ag} 1-\mathrm{Ag} 2$

I1-Ag1-Ag2

I2-Ag1-Ag2

P6-Ag1-Ag3

P1-Ag1-Ag3

I1-Ag1-Ag3

I2-Ag1-Ag3

$\mathrm{Ag} 2-\mathrm{Ag} 1-\mathrm{Ag} 3$

P3-Ag2-P2

P3-Ag2-I1

P2-Ag2-I1

P3-Ag2-I2

P2-Ag2-I2

I1-Ag2-I2

$\mathrm{P} 3-\mathrm{Ag} 2-\mathrm{Ag} 1$
$1.377(6)$

$1.390(7)$

$1.379(8)$

0.9300

$1.384(9)$

0.9300

$1.356(8)$

0.9300

1.374 (8)

0.9300

0.9300

$1.843(5)$

0.9700

0.9700

1.821 (13)

$1.823(5)$

$1.852(15)$

$1.375(7)$

1.389 (7)

$63.383(11)$

$65.867(12)$

$67.235(11)$

65.257 (12)

$66.541(12)$

$61.620(10)$

117.77 (4)

112.01 (3)

107.39 (3)

100.87 (3)

112.89 (3)

105.217 (14)

$151.56(3)$

90.20 (3)

59.434 (11)

$60.377(11)$

88.81 (3)

153.41 (3)

$58.479(11)$

$57.334(12)$

$63.309(11)$

123.75 (4)

111.95 (3)

$102.45(3)$

$99.29(3)$

$116.00(3)$

101.661 (13)

146.55 (3)
$\mathrm{C} 72-\mathrm{C} 73$

$\mathrm{C} 72-\mathrm{H} 72$

$\mathrm{C} 73-\mathrm{C} 74$

$\mathrm{C} 73-\mathrm{H} 73$

C74-C75

C74-H74

C75-H75

C77-C78

C77-C 82

C78-C79

C78- 778

C79-C 80

C79-H79

$\mathrm{C} 80-\mathrm{C} 81$

$\mathrm{C} 80-\mathrm{H} 80$

C $81-\mathrm{C} 82$

C81-H81

C $82-\mathrm{H} 82$

$\mathrm{C} 45 \mathrm{~B}-\mathrm{P} 4-\mathrm{C} 39$

$\mathrm{C} 45 \mathrm{~B}-\mathrm{P} 4-\mathrm{C} 38$

$\mathrm{C} 39-\mathrm{P} 4-\mathrm{C} 38$

$\mathrm{C} 39-\mathrm{P} 4-\mathrm{C} 45$

$\mathrm{C} 38-\mathrm{P} 4-\mathrm{C} 45$

$\mathrm{C} 45 \mathrm{~B}-\mathrm{P} 4-\mathrm{Ag} 3$

C39-P4-Ag3

C38-P4-Ag3

$\mathrm{C} 45-\mathrm{P} 4-\mathrm{Ag} 3$

$\mathrm{C} 44-\mathrm{C} 39-\mathrm{C} 40$

$\mathrm{C} 44-\mathrm{C} 39-\mathrm{P} 4$

$\mathrm{C} 40-\mathrm{C} 39-\mathrm{P} 4$

$\mathrm{C} 39-\mathrm{C} 40-\mathrm{C} 41$

C39- $440-\mathrm{H} 40$

$\mathrm{C} 41-\mathrm{C} 40-\mathrm{H} 40$

$\mathrm{C} 42-\mathrm{C} 41-\mathrm{C} 40$

$\mathrm{C} 42-\mathrm{C} 41-\mathrm{H} 41$

$\mathrm{C} 40-\mathrm{C} 41-\mathrm{H} 41$

$\mathrm{C} 41-\mathrm{C} 42-\mathrm{C} 43$

$\mathrm{C} 41-\mathrm{C} 42-\mathrm{H} 42$

$\mathrm{C} 43-\mathrm{C} 42-\mathrm{H} 42$

$\mathrm{C} 42-\mathrm{C} 43-\mathrm{C} 44$

$\mathrm{C} 42-\mathrm{C} 43-\mathrm{H} 43$

$\mathrm{C} 44-\mathrm{C} 43-\mathrm{H} 43$

$\mathrm{C} 39-\mathrm{C} 44-\mathrm{C} 43$

C39-C44- 444

$\mathrm{C} 43-\mathrm{C} 44-\mathrm{H} 44$

$\mathrm{C} 50-\mathrm{C} 45-\mathrm{C} 46$
$1.360(10)$

0.9300

$1.348(10)$

0.9300

$1.392(9)$

0.9300

0.9300

$1.362(11)$

$1.369(10)$

1.348 (12)

0.9300

1.368 (14)

0.9300

1.327 (14)

0.9300

$1.380(13)$

0.9300

0.9300

$104.5(4)$

$101.4(6)$

$106.1(2)$

$104.5(5)$

$100.3(7)$

$115.3(5)$

$114.90(16)$

$113.25(15)$

$116.2(5)$

$119.3(5)$

117.8 (4)

122.9 (4)

$120.2(5)$

119.9

119.9

119.5 (6)

120.2

120.2

$121.1(5)$

119.5

119.5

119.5 (6)

120.2

120.2

120.4 (6)

119.8

119.8

118.8 (13) 


\begin{tabular}{|c|c|c|c|}
\hline $\mathrm{P} 2-\mathrm{Ag} 2-\mathrm{Ag} 1$ & $89.53(3)$ & $\mathrm{C} 50-\mathrm{C} 45-\mathrm{P} 4$ & $118.7(11)$ \\
\hline $\mathrm{I} 1-\mathrm{Ag} 2-\mathrm{Ag} 1$ & $57.183(11)$ & $\mathrm{C} 46-\mathrm{C} 45-\mathrm{P} 4$ & $122.4(11)$ \\
\hline $\mathrm{I} 2-\mathrm{Ag} 2-\mathrm{Ag} 1$ & $58.003(11)$ & $\mathrm{C} 45-\mathrm{C} 46-\mathrm{C} 47$ & $121.2(13)$ \\
\hline $\mathrm{P} 3-\mathrm{Ag} 2-\mathrm{Ag} 3$ & $87.10(3)$ & $\mathrm{C} 45-\mathrm{C} 46-\mathrm{H} 46$ & 119.4 \\
\hline $\mathrm{P} 2-\mathrm{Ag} 2-\mathrm{Ag} 3$ & $148.86(3)$ & $\mathrm{C} 47-\mathrm{C} 46-\mathrm{H} 46$ & 119.4 \\
\hline $\mathrm{I} 1-\mathrm{Ag} 2-\mathrm{Ag} 3$ & $56.713(11)$ & $\mathrm{C} 48-\mathrm{C} 47-\mathrm{C} 46$ & $118.8(14)$ \\
\hline $\mathrm{I} 2-\mathrm{Ag} 2-\mathrm{Ag} 3$ & $55.614(11)$ & $\mathrm{C} 48-\mathrm{C} 47-\mathrm{H} 47$ & 120.6 \\
\hline $\mathrm{Ag} 1-\mathrm{Ag} 2-\mathrm{Ag} 3$ & $60.124(11)$ & $\mathrm{C} 46-\mathrm{C} 47-\mathrm{H} 47$ & 120.6 \\
\hline $\mathrm{P} 4-\mathrm{Ag} 3-\mathrm{P} 5$ & $127.78(4)$ & $\mathrm{C} 47-\mathrm{C} 48-\mathrm{C} 49$ & $122.2(14)$ \\
\hline P4-Ag3-I2 & $106.61(3)$ & $\mathrm{C} 47-\mathrm{C} 48-\mathrm{H} 48$ & 118.9 \\
\hline $\mathrm{P} 5-\mathrm{Ag} 3-\mathrm{I} 2$ & $98.37(3)$ & $\mathrm{C} 49-\mathrm{C} 48-\mathrm{H} 48$ & 118.9 \\
\hline P4-Ag3-I1 & $105.83(3)$ & $\mathrm{C} 48-\mathrm{C} 49-\mathrm{C} 50$ & $117.6(14)$ \\
\hline $\mathrm{P} 5-\mathrm{Ag} 3-\mathrm{I} 1$ & $112.23(3)$ & $\mathrm{C} 48-\mathrm{C} 49-\mathrm{H} 49$ & 121.2 \\
\hline $\mathrm{I} 2-\mathrm{Ag} 3-\mathrm{I} 1$ & $102.950(14)$ & $\mathrm{C} 50-\mathrm{C} 49-\mathrm{H} 49$ & 121.2 \\
\hline $\mathrm{P} 4-\mathrm{Ag} 3-\mathrm{Ag} 1$ & $144.31(3)$ & $\mathrm{C} 45-\mathrm{C} 50-\mathrm{C} 49$ & $121.3(14)$ \\
\hline P5-Ag3-Ag1 & $87.64(3)$ & $\mathrm{C} 45-\mathrm{C} 50-\mathrm{H} 50$ & 119.3 \\
\hline $\mathrm{I} 2-\mathrm{Ag} 3-\mathrm{Ag} 1$ & $57.409(11)$ & $\mathrm{C} 49-\mathrm{C} 50-\mathrm{H} 50$ & 119.3 \\
\hline I1-Ag3-Ag1 & $55.654(11)$ & $\mathrm{C} 46 \mathrm{~B}-\mathrm{C} 45 \mathrm{~B}-\mathrm{C} 50 \mathrm{~B}$ & $117.9(12)$ \\
\hline $\mathrm{P} 4-\mathrm{Ag} 3-\mathrm{Ag} 2$ & $87.76(3)$ & $\mathrm{C} 46 \mathrm{~B}-\mathrm{C} 45 \mathrm{~B}-\mathrm{P} 4$ & $122.8(10)$ \\
\hline P5-Ag3-Ag2 & $143.41(3)$ & $\mathrm{C} 50 \mathrm{~B}-\mathrm{C} 45 \mathrm{~B}-\mathrm{P} 4$ & $119.1(9)$ \\
\hline $\mathrm{I} 2-\mathrm{Ag} 3-\mathrm{Ag} 2$ & $57.845(11)$ & $\mathrm{C} 45 \mathrm{~B}-\mathrm{C} 46 \mathrm{~B}-\mathrm{C} 47 \mathrm{~B}$ & $121.1(12)$ \\
\hline $\mathrm{I} 1-\mathrm{Ag} 3-\mathrm{Ag} 2$ & $56.051(11)$ & $\mathrm{C} 45 \mathrm{~B}-\mathrm{C} 46 \mathrm{~B}-\mathrm{H} 46 \mathrm{~B}$ & 119.4 \\
\hline $\mathrm{Ag} 1-\mathrm{Ag} 3-\mathrm{Ag} 2$ & $56.567(10)$ & $\mathrm{C} 47 \mathrm{~B}-\mathrm{C} 46 \mathrm{~B}-\mathrm{H} 46 \mathrm{~B}$ & 119.4 \\
\hline $\mathrm{C} 76-\mathrm{N} 1-\mathrm{H} 1 \mathrm{~A}$ & 120.0 & $\mathrm{C} 48 \mathrm{~B}-\mathrm{C} 47 \mathrm{~B}-\mathrm{C} 46 \mathrm{~B}$ & $120.0(13)$ \\
\hline $\mathrm{C} 76-\mathrm{N} 1-\mathrm{H} 1 \mathrm{~B}$ & 120.0 & $\mathrm{C} 48 \mathrm{~B}-\mathrm{C} 47 \mathrm{~B}-\mathrm{H} 47 \mathrm{~B}$ & 120.0 \\
\hline $\mathrm{H} 1 \mathrm{~A}-\mathrm{N} 1-\mathrm{H} 1 \mathrm{~B}$ & 120.0 & $\mathrm{C} 46 \mathrm{~B}-\mathrm{C} 47 \mathrm{~B}-\mathrm{H} 47 \mathrm{~B}$ & 120.0 \\
\hline $\mathrm{C} 76-\mathrm{N} 2-\mathrm{C} 77$ & $129.6(7)$ & $\mathrm{C} 47 \mathrm{~B}-\mathrm{C} 48 \mathrm{~B}-\mathrm{C} 49 \mathrm{~B}$ & $120.8(13)$ \\
\hline $\mathrm{C} 76-\mathrm{N} 2-\mathrm{H} 2 \mathrm{~A}$ & 115.2 & $\mathrm{C} 47 \mathrm{~B}-\mathrm{C} 48 \mathrm{~B}-\mathrm{H} 48 \mathrm{~B}$ & 119.6 \\
\hline $\mathrm{C} 77-\mathrm{N} 2-\mathrm{H} 2 \mathrm{~A}$ & 115.2 & $\mathrm{C} 49 \mathrm{~B}-\mathrm{C} 48 \mathrm{~B}-\mathrm{H} 48 \mathrm{~B}$ & 119.6 \\
\hline $\mathrm{C} 7-\mathrm{P} 1-\mathrm{C} 1$ & $106.0(2)$ & $\mathrm{C} 48 \mathrm{~B}-\mathrm{C} 49 \mathrm{~B}-\mathrm{C} 50 \mathrm{~B}$ & $118.1(12)$ \\
\hline $\mathrm{C} 7-\mathrm{P} 1-\mathrm{C} 13$ & $106.9(2)$ & $\mathrm{C} 48 \mathrm{~B}-\mathrm{C} 49 \mathrm{~B}-\mathrm{H} 49 \mathrm{~B}$ & 120.9 \\
\hline $\mathrm{C} 1-\mathrm{P} 1-\mathrm{C} 13$ & $101.2(2)$ & $\mathrm{C} 50 \mathrm{~B}-\mathrm{C} 49 \mathrm{~B}-\mathrm{H} 49 \mathrm{~B}$ & 120.9 \\
\hline $\mathrm{C} 7-\mathrm{P} 1-\mathrm{Ag} 1$ & $117.36(15)$ & $\mathrm{C} 45 \mathrm{~B}-\mathrm{C} 50 \mathrm{~B}-\mathrm{C} 49 \mathrm{~B}$ & $121.9(12)$ \\
\hline $\mathrm{C} 1-\mathrm{P} 1-\mathrm{Ag} 1$ & $111.52(17)$ & $\mathrm{C} 45 \mathrm{~B}-\mathrm{C} 50 \mathrm{~B}-\mathrm{H} 50 \mathrm{~B}$ & 119.1 \\
\hline $\mathrm{C} 13-\mathrm{P} 1-\mathrm{Ag} 1$ & $112.43(16)$ & $\mathrm{C} 49 \mathrm{~B}-\mathrm{C} 50 \mathrm{~B}-\mathrm{H} 50 \mathrm{~B}$ & 119.1 \\
\hline $\mathrm{C} 6-\mathrm{C} 1-\mathrm{C} 2$ & $118.3(5)$ & $\mathrm{C} 51 \mathrm{~B}-\mathrm{P} 5-\mathrm{C} 57$ & $103.3(5)$ \\
\hline $\mathrm{C} 6-\mathrm{C} 1-\mathrm{P} 1$ & $124.9(4)$ & $\mathrm{C} 51 \mathrm{~B}-\mathrm{P} 5-\mathrm{C} 63$ & $100.2(6)$ \\
\hline $\mathrm{C} 2-\mathrm{C} 1-\mathrm{P} 1$ & $116.8(4)$ & $\mathrm{C} 57-\mathrm{P} 5-\mathrm{C} 63$ & $106.0(3)$ \\
\hline $\mathrm{C} 1-\mathrm{C} 2-\mathrm{C} 3$ & $120.6(6)$ & $\mathrm{C} 57-\mathrm{P} 5-\mathrm{C} 51$ & $103.2(7)$ \\
\hline $\mathrm{C} 1-\mathrm{C} 2-\mathrm{H} 2$ & 119.7 & $\mathrm{C} 63-\mathrm{P} 5-\mathrm{C} 51$ & $105.1(7)$ \\
\hline $\mathrm{C} 3-\mathrm{C} 2-\mathrm{H} 2$ & 119.7 & $\mathrm{C} 51 \mathrm{~B}-\mathrm{P} 5-\mathrm{Ag} 3$ & $112.7(6)$ \\
\hline $\mathrm{C} 4-\mathrm{C} 3-\mathrm{C} 2$ & $119.8(7)$ & $\mathrm{C} 57-\mathrm{P} 5-\mathrm{Ag} 3$ & $118.11(19)$ \\
\hline $\mathrm{C} 4-\mathrm{C} 3-\mathrm{H} 3$ & 120.1 & $\mathrm{C} 63-\mathrm{P} 5-\mathrm{Ag} 3$ & $114.48(16)$ \\
\hline $\mathrm{C} 2-\mathrm{C} 3-\mathrm{H} 3$ & 120.1 & $\mathrm{C} 51-\mathrm{P} 5-\mathrm{Ag} 3$ & $108.6(8)$ \\
\hline $\mathrm{C} 5-\mathrm{C} 4-\mathrm{C} 3$ & $119.8(6)$ & $\mathrm{C} 52-\mathrm{C} 51-\mathrm{C} 56$ & $118.8(14)$ \\
\hline $\mathrm{C} 5-\mathrm{C} 4-\mathrm{H} 4$ & 120.1 & $\mathrm{C} 52-\mathrm{C} 51-\mathrm{P} 5$ & $125.0(12)$ \\
\hline $\mathrm{C} 3-\mathrm{C} 4-\mathrm{H} 4$ & 120.1 & $\mathrm{C} 56-\mathrm{C} 51-\mathrm{P} 5$ & $116.1(13)$ \\
\hline $\mathrm{C} 4-\mathrm{C} 5-\mathrm{C} 6$ & $121.1(7)$ & $\mathrm{C} 51-\mathrm{C} 52-\mathrm{C} 53$ & $120.6(14)$ \\
\hline
\end{tabular}




\begin{tabular}{|c|c|c|c|}
\hline $\mathrm{C} 4-\mathrm{C} 5-\mathrm{H} 5$ & 119.4 & $\mathrm{C} 51-\mathrm{C} 52-\mathrm{H} 52$ & 119.7 \\
\hline $\mathrm{C} 6-\mathrm{C} 5-\mathrm{H} 5$ & 119.4 & $\mathrm{C} 53-\mathrm{C} 52-\mathrm{H} 52$ & 119.7 \\
\hline $\mathrm{C} 1-\mathrm{C} 6-\mathrm{C} 5$ & $120.3(7)$ & $\mathrm{C} 54-\mathrm{C} 53-\mathrm{C} 52$ & $119.7(15)$ \\
\hline $\mathrm{C} 1-\mathrm{C} 6-\mathrm{H} 6$ & 119.8 & $\mathrm{C} 54-\mathrm{C} 53-\mathrm{H} 53$ & 120.2 \\
\hline $\mathrm{C} 5-\mathrm{C} 6-\mathrm{H} 6$ & 119.8 & $\mathrm{C} 52-\mathrm{C} 53-\mathrm{H} 53$ & 120.2 \\
\hline $\mathrm{C} 8-\mathrm{C} 7-\mathrm{C} 12$ & $118.2(5)$ & $\mathrm{C} 55-\mathrm{C} 54-\mathrm{C} 53$ & $120.4(15)$ \\
\hline $\mathrm{C} 8-\mathrm{C} 7-\mathrm{P} 1$ & $117.5(4)$ & $\mathrm{C} 55-\mathrm{C} 54-\mathrm{H} 54$ & 119.8 \\
\hline $\mathrm{C} 12-\mathrm{C} 7-\mathrm{P} 1$ & $124.3(4)$ & $\mathrm{C} 53-\mathrm{C} 54-\mathrm{H} 54$ & 119.8 \\
\hline $\mathrm{C} 9-\mathrm{C} 8-\mathrm{C} 7$ & $121.2(5)$ & $\mathrm{C} 54-\mathrm{C} 55-\mathrm{C} 56$ & $121.4(15)$ \\
\hline $\mathrm{C} 9-\mathrm{C} 8-\mathrm{H} 8$ & 119.4 & C54-C55-H55 & 119.3 \\
\hline $\mathrm{C} 7-\mathrm{C} 8-\mathrm{H} 8$ & 119.4 & $\mathrm{C} 56-\mathrm{C} 55-\mathrm{H} 55$ & 119.3 \\
\hline $\mathrm{C} 10-\mathrm{C} 9-\mathrm{C} 8$ & $119.1(6)$ & $\mathrm{C} 55-\mathrm{C} 56-\mathrm{C} 51$ & $118.9(14)$ \\
\hline $\mathrm{C} 10-\mathrm{C} 9-\mathrm{H} 9$ & 120.5 & $\mathrm{C} 55-\mathrm{C} 56-\mathrm{H} 56$ & 120.6 \\
\hline $\mathrm{C} 8-\mathrm{C} 9-\mathrm{H} 9$ & 120.5 & C51-C56-H56 & 120.6 \\
\hline $\mathrm{C} 9-\mathrm{C} 10-\mathrm{C} 11$ & $121.4(6)$ & $\mathrm{C} 52 \mathrm{~B}-\mathrm{C} 51 \mathrm{~B}-\mathrm{C} 56 \mathrm{~B}$ & $120.9(12)$ \\
\hline $\mathrm{C} 9-\mathrm{C} 10-\mathrm{H} 10$ & 119.3 & $\mathrm{C} 52 \mathrm{~B}-\mathrm{C} 51 \mathrm{~B}-\mathrm{P} 5$ & $118.0(10)$ \\
\hline $\mathrm{C} 11-\mathrm{C} 10-\mathrm{H} 10$ & 119.3 & $\mathrm{C} 56 \mathrm{~B}-\mathrm{C} 51 \mathrm{~B}-\mathrm{P} 5$ & $121.1(11)$ \\
\hline $\mathrm{C} 10-\mathrm{C} 11-\mathrm{C} 12$ & $119.8(6)$ & $\mathrm{C} 51 \mathrm{~B}-\mathrm{C} 52 \mathrm{~B}-\mathrm{C} 53 \mathrm{~B}$ & $119.4(13)$ \\
\hline $\mathrm{C} 10-\mathrm{C} 11-\mathrm{H} 11$ & 120.1 & $\mathrm{C} 51 \mathrm{~B}-\mathrm{C} 52 \mathrm{~B}-\mathrm{H} 52 \mathrm{~B}$ & 120.3 \\
\hline $\mathrm{C} 12-\mathrm{C} 11-\mathrm{H} 11$ & 120.1 & $\mathrm{C} 53 \mathrm{~B}-\mathrm{C} 52 \mathrm{~B}-\mathrm{H} 52 \mathrm{~B}$ & 120.3 \\
\hline $\mathrm{C} 11-\mathrm{C} 12-\mathrm{C} 7$ & $120.3(5)$ & $\mathrm{C} 54 \mathrm{~B}-\mathrm{C} 53 \mathrm{~B}-\mathrm{C} 52 \mathrm{~B}$ & $119.2(13)$ \\
\hline $\mathrm{C} 11-\mathrm{C} 12-\mathrm{H} 12$ & 119.8 & $\mathrm{C} 54 \mathrm{~B}-\mathrm{C} 53 \mathrm{~B}-\mathrm{H} 53 \mathrm{~B}$ & 120.4 \\
\hline $\mathrm{C} 7-\mathrm{C} 12-\mathrm{H} 12$ & 119.8 & $\mathrm{C} 52 \mathrm{~B}-\mathrm{C} 53 \mathrm{~B}-\mathrm{H} 53 \mathrm{~B}$ & 120.4 \\
\hline $\mathrm{P} 1-\mathrm{C} 13-\mathrm{P} 2$ & $113.7(2)$ & $\mathrm{C} 55 \mathrm{~B}-\mathrm{C} 54 \mathrm{~B}-\mathrm{C} 53 \mathrm{~B}$ & $122.3(12)$ \\
\hline $\mathrm{P} 1-\mathrm{C} 13-\mathrm{H} 13 \mathrm{~A}$ & 108.8 & $\mathrm{C} 55 \mathrm{~B}-\mathrm{C} 54 \mathrm{~B}-\mathrm{H} 54 \mathrm{~B}$ & 118.9 \\
\hline $\mathrm{P} 2-\mathrm{C} 13-\mathrm{H} 13 \mathrm{~A}$ & 108.8 & $\mathrm{C} 53 \mathrm{~B}-\mathrm{C} 54 \mathrm{~B}-\mathrm{H} 54 \mathrm{~B}$ & 118.9 \\
\hline $\mathrm{P} 1-\mathrm{C} 13-\mathrm{H} 13 \mathrm{~B}$ & 108.8 & $\mathrm{C} 54 \mathrm{~B}-\mathrm{C} 55 \mathrm{~B}-\mathrm{C} 56 \mathrm{~B}$ & $120.1(13)$ \\
\hline $\mathrm{P} 2-\mathrm{C} 13-\mathrm{H} 13 \mathrm{~B}$ & 108.8 & $\mathrm{C} 54 \mathrm{~B}-\mathrm{C} 55 \mathrm{~B}-\mathrm{H} 55 \mathrm{~B}$ & 120.0 \\
\hline $\mathrm{H} 13 \mathrm{~A}-\mathrm{C} 13-\mathrm{H} 13 \mathrm{~B}$ & 107.7 & $\mathrm{C} 56 \mathrm{~B}-\mathrm{C} 55 \mathrm{~B}-\mathrm{H} 55 \mathrm{~B}$ & 120.0 \\
\hline $\mathrm{C} 14-\mathrm{P} 2-\mathrm{C} 20$ & $107.4(2)$ & $\mathrm{C} 51 \mathrm{~B}-\mathrm{C} 56 \mathrm{~B}-\mathrm{C} 55 \mathrm{~B}$ & $118.2(13)$ \\
\hline $\mathrm{C} 14-\mathrm{P} 2-\mathrm{C} 13$ & $104.7(2)$ & $\mathrm{C} 51 \mathrm{~B}-\mathrm{C} 56 \mathrm{~B}-\mathrm{H} 56 \mathrm{~B}$ & 120.9 \\
\hline $\mathrm{C} 20-\mathrm{P} 2-\mathrm{C} 13$ & $101.0(2)$ & $\mathrm{C} 55 \mathrm{~B}-\mathrm{C} 56 \mathrm{~B}-\mathrm{H} 56 \mathrm{~B}$ & 120.9 \\
\hline $\mathrm{C} 14-\mathrm{P} 2-\mathrm{Ag} 2$ & $115.15(15)$ & $\mathrm{C} 58-\mathrm{C} 57-\mathrm{C} 62$ & $118.4(6)$ \\
\hline $\mathrm{C} 20-\mathrm{P} 2-\mathrm{Ag} 2$ & $112.91(17)$ & $\mathrm{C} 58-\mathrm{C} 57-\mathrm{P} 5$ & $118.8(5)$ \\
\hline $\mathrm{C} 13-\mathrm{P} 2-\mathrm{Ag} 2$ & $114.36(15)$ & $\mathrm{C} 62-\mathrm{C} 57-\mathrm{P} 5$ & $122.7(5)$ \\
\hline $\mathrm{C} 19-\mathrm{C} 14-\mathrm{C} 15$ & $118.5(5)$ & $\mathrm{C} 57-\mathrm{C} 58-\mathrm{C} 59$ & $121.7(8)$ \\
\hline $\mathrm{C} 19-\mathrm{C} 14-\mathrm{P} 2$ & $124.2(4)$ & $\mathrm{C} 57-\mathrm{C} 58-\mathrm{H} 58$ & 119.2 \\
\hline $\mathrm{C} 15-\mathrm{C} 14-\mathrm{P} 2$ & $117.3(4)$ & $\mathrm{C} 59-\mathrm{C} 58-\mathrm{H} 58$ & 119.2 \\
\hline $\mathrm{C} 16-\mathrm{C} 15-\mathrm{C} 14$ & $120.1(5)$ & $\mathrm{C} 60-\mathrm{C} 59-\mathrm{C} 58$ & $118.6(9)$ \\
\hline $\mathrm{C} 16-\mathrm{C} 15-\mathrm{H} 15$ & 120.0 & $\mathrm{C} 60-\mathrm{C} 59-\mathrm{H} 59$ & 120.7 \\
\hline $\mathrm{C} 14-\mathrm{C} 15-\mathrm{H} 15$ & 120.0 & $\mathrm{C} 58-\mathrm{C} 59-\mathrm{H} 59$ & 120.7 \\
\hline $\mathrm{C} 17-\mathrm{C} 16-\mathrm{C} 15$ & $120.8(6)$ & $\mathrm{C} 59-\mathrm{C} 60-\mathrm{C} 61$ & $120.5(8)$ \\
\hline $\mathrm{C} 17-\mathrm{C} 16-\mathrm{H} 16$ & 119.6 & $\mathrm{C} 59-\mathrm{C} 60-\mathrm{H} 60$ & 119.8 \\
\hline $\mathrm{C} 15-\mathrm{C} 16-\mathrm{H} 16$ & 119.6 & $\mathrm{C} 61-\mathrm{C} 60-\mathrm{H} 60$ & 119.8 \\
\hline $\mathrm{C} 16-\mathrm{C} 17-\mathrm{C} 18$ & $119.4(6)$ & $\mathrm{C} 62-\mathrm{C} 61-\mathrm{C} 60$ & $120.2(9)$ \\
\hline $\mathrm{C} 16-\mathrm{C} 17-\mathrm{H} 17$ & 120.3 & $\mathrm{C} 62-\mathrm{C} 61-\mathrm{H} 61$ & 119.9 \\
\hline $\mathrm{C} 18-\mathrm{C} 17-\mathrm{H} 17$ & 120.3 & $\mathrm{C} 60-\mathrm{C} 61-\mathrm{H} 61$ & 119.9 \\
\hline $\mathrm{C} 17-\mathrm{C} 18-\mathrm{C} 19$ & $120.7(6)$ & $\mathrm{C} 61-\mathrm{C} 62-\mathrm{C} 57$ & $120.3(8)$ \\
\hline
\end{tabular}




\begin{tabular}{|c|c|c|c|}
\hline $\mathrm{C} 17-\mathrm{C} 18-\mathrm{H} 18$ & 119.7 & $\mathrm{C} 61-\mathrm{C} 62-\mathrm{H} 62$ & 119.8 \\
\hline $\mathrm{C} 19-\mathrm{C} 18-\mathrm{H} 18$ & 119.7 & C57-C62-H62 & 119.8 \\
\hline $\mathrm{C} 18-\mathrm{C} 19-\mathrm{C} 14$ & $120.6(6)$ & $\mathrm{P} 6-\mathrm{C} 63-\mathrm{P} 5$ & $112.3(3)$ \\
\hline $\mathrm{C} 18-\mathrm{C} 19-\mathrm{H} 19$ & 119.7 & $\mathrm{P} 6-\mathrm{C} 63-\mathrm{H} 63 \mathrm{~A}$ & 109.1 \\
\hline $\mathrm{C} 14-\mathrm{C} 19-\mathrm{H} 19$ & 119.7 & $\mathrm{P} 5-\mathrm{C} 63-\mathrm{H} 63 \mathrm{~A}$ & 109.1 \\
\hline $\mathrm{C} 21-\mathrm{C} 20-\mathrm{C} 25$ & $118.3(5)$ & $\mathrm{P} 6-\mathrm{C} 63-\mathrm{H} 63 \mathrm{~B}$ & 109.1 \\
\hline $\mathrm{C} 21-\mathrm{C} 20-\mathrm{P} 2$ & $123.4(4)$ & $\mathrm{P} 5-\mathrm{C} 63-\mathrm{H} 63 \mathrm{~B}$ & 109.1 \\
\hline $\mathrm{C} 25-\mathrm{C} 20-\mathrm{P} 2$ & $118.1(4)$ & $\mathrm{H} 63 \mathrm{~A}-\mathrm{C} 63-\mathrm{H} 63 \mathrm{~B}$ & 107.9 \\
\hline $\mathrm{C} 22-\mathrm{C} 21-\mathrm{C} 20$ & $121.2(6)$ & $\mathrm{C} 64-\mathrm{P} 6-\mathrm{C} 70$ & $102.2(2)$ \\
\hline $\mathrm{C} 22-\mathrm{C} 21-\mathrm{H} 21$ & 119.4 & $\mathrm{C} 64-\mathrm{P} 6-\mathrm{C} 63$ & $104.9(2)$ \\
\hline $\mathrm{C} 20-\mathrm{C} 21-\mathrm{H} 21$ & 119.4 & $\mathrm{C} 70-\mathrm{P} 6-\mathrm{C} 63$ & $105.7(2)$ \\
\hline $\mathrm{C} 21-\mathrm{C} 22-\mathrm{C} 23$ & $119.9(6)$ & $\mathrm{C} 64-\mathrm{P} 6-\mathrm{Ag} 1$ & $122.41(17)$ \\
\hline $\mathrm{C} 21-\mathrm{C} 22-\mathrm{H} 22$ & 120.1 & $\mathrm{C} 70-\mathrm{P} 6-\mathrm{Ag} 1$ & $107.26(15)$ \\
\hline $\mathrm{C} 23-\mathrm{C} 22-\mathrm{H} 22$ & 120.1 & $\mathrm{C} 63-\mathrm{P} 6-\mathrm{Ag} 1$ & $112.84(16)$ \\
\hline $\mathrm{C} 24-\mathrm{C} 23-\mathrm{C} 22$ & $119.8(6)$ & $\mathrm{C} 65-\mathrm{C} 64-\mathrm{C} 69$ & $119.0(5)$ \\
\hline $\mathrm{C} 24-\mathrm{C} 23-\mathrm{H} 23$ & 120.1 & $\mathrm{C} 65-\mathrm{C} 64-\mathrm{P} 6$ & $119.7(4)$ \\
\hline $\mathrm{C} 22-\mathrm{C} 23-\mathrm{H} 23$ & 120.1 & $\mathrm{C} 69-\mathrm{C} 64-\mathrm{P} 6$ & $121.2(4)$ \\
\hline $\mathrm{C} 23-\mathrm{C} 24-\mathrm{C} 25$ & $120.5(6)$ & $\mathrm{C} 64-\mathrm{C} 65-\mathrm{C} 66$ & $119.8(5)$ \\
\hline $\mathrm{C} 23-\mathrm{C} 24-\mathrm{H} 24$ & 119.7 & $\mathrm{C} 64-\mathrm{C} 65-\mathrm{H} 65$ & 120.1 \\
\hline $\mathrm{C} 25-\mathrm{C} 24-\mathrm{H} 24$ & 119.7 & C66-C65-H65 & 120.1 \\
\hline $\mathrm{C} 20-\mathrm{C} 25-\mathrm{C} 24$ & $120.2(6)$ & $\mathrm{C} 67-\mathrm{C} 66-\mathrm{C} 65$ & $120.5(6)$ \\
\hline $\mathrm{C} 20-\mathrm{C} 25-\mathrm{H} 25$ & 119.9 & C67-C66-H66 & 119.8 \\
\hline $\mathrm{C} 24-\mathrm{C} 25-\mathrm{H} 25$ & 119.9 & $\mathrm{C} 65-\mathrm{C} 66-\mathrm{H} 66$ & 119.8 \\
\hline $\mathrm{C} 26-\mathrm{P} 3-\mathrm{C} 32$ & $104.3(2)$ & $\mathrm{C} 66-\mathrm{C} 67-\mathrm{C} 68$ & $120.7(6)$ \\
\hline $\mathrm{C} 26-\mathrm{P} 3-\mathrm{C} 38$ & $103.0(2)$ & C66-C67-H67 & 119.7 \\
\hline $\mathrm{C} 32-\mathrm{P} 3-\mathrm{C} 38$ & $105.8(2)$ & C68-C67-H67 & 119.7 \\
\hline $\mathrm{C} 26-\mathrm{P} 3-\mathrm{Ag} 2$ & $111.72(15)$ & $\mathrm{C} 67-\mathrm{C} 68-\mathrm{C} 69$ & $119.7(6)$ \\
\hline $\mathrm{C} 32-\mathrm{P} 3-\mathrm{Ag} 2$ & $113.43(14)$ & C67-C68-H68 & 120.1 \\
\hline $\mathrm{C} 38-\mathrm{P} 3-\mathrm{Ag} 2$ & $117.28(15)$ & $\mathrm{C} 69-\mathrm{C} 68-\mathrm{H} 68$ & 120.1 \\
\hline $\mathrm{C} 27-\mathrm{C} 26-\mathrm{C} 31$ & $118.3(4)$ & С68-C69-C64 & $120.2(6)$ \\
\hline $\mathrm{C} 27-\mathrm{C} 26-\mathrm{P} 3$ & $117.9(3)$ & C68-C69-H69 & 119.9 \\
\hline $\mathrm{C} 31-\mathrm{C} 26-\mathrm{P} 3$ & $123.8(4)$ & C64-C69-H69 & 119.9 \\
\hline $\mathrm{C} 28-\mathrm{C} 27-\mathrm{C} 26$ & $120.7(5)$ & $\mathrm{C} 71-\mathrm{C} 70-\mathrm{C} 75$ & $118.5(5)$ \\
\hline $\mathrm{C} 28-\mathrm{C} 27-\mathrm{H} 27$ & 119.6 & $\mathrm{C} 71-\mathrm{C} 70-\mathrm{P} 6$ & $124.3(4)$ \\
\hline $\mathrm{C} 26-\mathrm{C} 27-\mathrm{H} 27$ & 119.6 & $\mathrm{C} 75-\mathrm{C} 70-\mathrm{P} 6$ & $116.8(4)$ \\
\hline $\mathrm{C} 29-\mathrm{C} 28-\mathrm{C} 27$ & $120.7(5)$ & $\mathrm{C} 70-\mathrm{C} 71-\mathrm{C} 72$ & $120.2(6)$ \\
\hline $\mathrm{C} 29-\mathrm{C} 28-\mathrm{H} 28$ & 119.7 & $\mathrm{C} 70-\mathrm{C} 71-\mathrm{H} 71$ & 119.9 \\
\hline $\mathrm{C} 27-\mathrm{C} 28-\mathrm{H} 28$ & 119.7 & $\mathrm{C} 72-\mathrm{C} 71-\mathrm{H} 71$ & 119.9 \\
\hline $\mathrm{C} 28-\mathrm{C} 29-\mathrm{C} 30$ & $119.6(5)$ & $\mathrm{C} 73-\mathrm{C} 72-\mathrm{C} 71$ & $121.0(6)$ \\
\hline $\mathrm{C} 28-\mathrm{C} 29-\mathrm{H} 29$ & 120.2 & $\mathrm{C} 73-\mathrm{C} 72-\mathrm{H} 72$ & 119.5 \\
\hline $\mathrm{C} 30-\mathrm{C} 29-\mathrm{H} 29$ & 120.2 & $\mathrm{C} 71-\mathrm{C} 72-\mathrm{H} 72$ & 119.5 \\
\hline $\mathrm{C} 29-\mathrm{C} 30-\mathrm{C} 31$ & $120.9(5)$ & $\mathrm{C} 74-\mathrm{C} 73-\mathrm{C} 72$ & $119.7(6)$ \\
\hline $\mathrm{C} 29-\mathrm{C} 30-\mathrm{H} 30$ & 119.6 & $\mathrm{C} 74-\mathrm{C} 73-\mathrm{H} 73$ & 120.2 \\
\hline $\mathrm{C} 31-\mathrm{C} 30-\mathrm{H} 30$ & 119.6 & $\mathrm{C} 72-\mathrm{C} 73-\mathrm{H} 73$ & 120.2 \\
\hline $\mathrm{C} 30-\mathrm{C} 31-\mathrm{C} 26$ & $119.9(5)$ & $\mathrm{C} 73-\mathrm{C} 74-\mathrm{C} 75$ & $120.4(6)$ \\
\hline $\mathrm{C} 30-\mathrm{C} 31-\mathrm{H} 31$ & 120.1 & $\mathrm{C} 73-\mathrm{C} 74-\mathrm{H} 74$ & 119.8 \\
\hline $\mathrm{C} 26-\mathrm{C} 31-\mathrm{H} 31$ & 120.1 & $\mathrm{C} 75-\mathrm{C} 74-\mathrm{H} 74$ & 119.8 \\
\hline $\mathrm{C} 37-\mathrm{C} 32-\mathrm{C} 33$ & $118.8(4)$ & $\mathrm{C} 70-\mathrm{C} 75-\mathrm{C} 74$ & $120.2(6)$ \\
\hline
\end{tabular}




\begin{tabular}{|c|c|c|c|}
\hline $\mathrm{C} 37-\mathrm{C} 32-\mathrm{P} 3$ & $118.2(3)$ & $\mathrm{C} 70-\mathrm{C} 75-\mathrm{H} 75$ & 119.9 \\
\hline $\mathrm{C} 33-\mathrm{C} 32-\mathrm{P} 3$ & $122.9(4)$ & $\mathrm{C} 74-\mathrm{C} 75-\mathrm{H} 75$ & 119.9 \\
\hline $\mathrm{C} 34-\mathrm{C} 33-\mathrm{C} 32$ & $119.9(5)$ & $\mathrm{N} 2-\mathrm{C} 76-\mathrm{N} 1$ & $111.2(8)$ \\
\hline $\mathrm{C} 34-\mathrm{C} 33-\mathrm{H} 33$ & 120.0 & $\mathrm{~N} 2-\mathrm{C} 76-\mathrm{S} 1$ & $127.4(7)$ \\
\hline $\mathrm{C} 32-\mathrm{C} 33-\mathrm{H} 33$ & 120.0 & $\mathrm{~N} 1-\mathrm{C} 76-\mathrm{S} 1$ & $121.4(8)$ \\
\hline $\mathrm{C} 33-\mathrm{C} 34-\mathrm{C} 35$ & $120.2(5)$ & $\mathrm{C} 78-\mathrm{C} 77-\mathrm{C} 82$ & $119.3(8)$ \\
\hline $\mathrm{C} 33-\mathrm{C} 34-\mathrm{H} 34$ & 119.9 & $\mathrm{C} 78-\mathrm{C} 77-\mathrm{N} 2$ & $116.5(7)$ \\
\hline $\mathrm{C} 35-\mathrm{C} 34-\mathrm{H} 34$ & 119.9 & $\mathrm{C} 82-\mathrm{C} 77-\mathrm{N} 2$ & $124.1(8)$ \\
\hline $\mathrm{C} 36-\mathrm{C} 35-\mathrm{C} 34$ & $119.6(5)$ & $\mathrm{C} 79-\mathrm{C} 78-\mathrm{C} 77$ & $121.2(9)$ \\
\hline $\mathrm{C} 36-\mathrm{C} 35-\mathrm{H} 35$ & 120.2 & $\mathrm{C} 79-\mathrm{C} 78-\mathrm{H} 78$ & 119.4 \\
\hline $\mathrm{C} 34-\mathrm{C} 35-\mathrm{H} 35$ & 120.2 & $\mathrm{C} 77-\mathrm{C} 78-\mathrm{H} 78$ & 119.4 \\
\hline $\mathrm{C} 35-\mathrm{C} 36-\mathrm{C} 37$ & $120.8(5)$ & $\mathrm{C} 78-\mathrm{C} 79-\mathrm{C} 80$ & $119.8(10)$ \\
\hline $\mathrm{C} 35-\mathrm{C} 36-\mathrm{H} 36$ & 119.6 & $\mathrm{C} 78-\mathrm{C} 79-\mathrm{H} 79$ & 120.1 \\
\hline $\mathrm{C} 37-\mathrm{C} 36-\mathrm{H} 36$ & 119.6 & $\mathrm{C} 80-\mathrm{C} 79-\mathrm{H} 79$ & 120.1 \\
\hline $\mathrm{C} 36-\mathrm{C} 37-\mathrm{C} 32$ & $120.6(5)$ & $\mathrm{C} 81-\mathrm{C} 80-\mathrm{C} 79$ & $119.5(10)$ \\
\hline $\mathrm{C} 36-\mathrm{C} 37-\mathrm{H} 37$ & 119.7 & $\mathrm{C} 81-\mathrm{C} 80-\mathrm{H} 80$ & 120.3 \\
\hline $\mathrm{C} 32-\mathrm{C} 37-\mathrm{H} 37$ & 119.7 & $\mathrm{C} 79-\mathrm{C} 80-\mathrm{H} 80$ & 120.3 \\
\hline $\mathrm{P} 4-\mathrm{C} 38-\mathrm{P} 3$ & $114.2(2)$ & $\mathrm{C} 80-\mathrm{C} 81-\mathrm{C} 82$ & $121.9(10)$ \\
\hline $\mathrm{P} 4-\mathrm{C} 38-\mathrm{H} 38 \mathrm{~A}$ & 108.7 & $\mathrm{C} 80-\mathrm{C} 81-\mathrm{H} 81$ & 119.0 \\
\hline $\mathrm{P} 3-\mathrm{C} 38-\mathrm{H} 38 \mathrm{~A}$ & 108.7 & $\mathrm{C} 82-\mathrm{C} 81-\mathrm{H} 81$ & 119.0 \\
\hline $\mathrm{P} 4-\mathrm{C} 38-\mathrm{H} 38 \mathrm{~B}$ & 108.7 & $\mathrm{C} 77-\mathrm{C} 82-\mathrm{C} 81$ & $118.3(9)$ \\
\hline $\mathrm{P} 3-\mathrm{C} 38-\mathrm{H} 38 \mathrm{~B}$ & 108.7 & $\mathrm{C} 77-\mathrm{C} 82-\mathrm{H} 82$ & 120.8 \\
\hline $\mathrm{H} 38 \mathrm{~A}-\mathrm{C} 38-\mathrm{H} 38 \mathrm{~B}$ & 107.6 & $\mathrm{C} 81-\mathrm{C} 82-\mathrm{H} 82$ & 120.8 \\
\hline $\mathrm{C} 7-\mathrm{P} 1-\mathrm{C} 1-\mathrm{C} 6$ & $-19.0(6)$ & $\mathrm{C} 39-\mathrm{P} 4-\mathrm{C} 45-\mathrm{C} 46$ & $-33.3(17)$ \\
\hline $\mathrm{C} 13-\mathrm{P} 1-\mathrm{C} 1-\mathrm{C} 6$ & $92.4(6)$ & $\mathrm{C} 38-\mathrm{P} 4-\mathrm{C} 45-\mathrm{C} 46$ & $76.5(16)$ \\
\hline $\mathrm{Ag} 1-\mathrm{P} 1-\mathrm{C} 1-\mathrm{C} 6$ & $-147.9(6)$ & $\mathrm{Ag} 3-\mathrm{P} 4-\mathrm{C} 45-\mathrm{C} 46$ & $-161.1(13)$ \\
\hline $\mathrm{C} 7-\mathrm{P} 1-\mathrm{C} 1-\mathrm{C} 2$ & $159.3(5)$ & $\mathrm{C} 50-\mathrm{C} 45-\mathrm{C} 46-\mathrm{C} 47$ & $-1.5(19)$ \\
\hline $\mathrm{C} 13-\mathrm{P} 1-\mathrm{C} 1-\mathrm{C} 2$ & $-89.3(5)$ & $\mathrm{P} 4-\mathrm{C} 45-\mathrm{C} 46-\mathrm{C} 47$ & $-176.6(16)$ \\
\hline $\mathrm{Ag} 1-\mathrm{P} 1-\mathrm{C} 1-\mathrm{C} 2$ & $30.4(5)$ & $\mathrm{C} 45-\mathrm{C} 46-\mathrm{C} 47-\mathrm{C} 48$ & $1(2)$ \\
\hline $\mathrm{C} 6-\mathrm{C} 1-\mathrm{C} 2-\mathrm{C} 3$ & $1.6(11)$ & $\mathrm{C} 46-\mathrm{C} 47-\mathrm{C} 48-\mathrm{C} 49$ & $-1(3)$ \\
\hline $\mathrm{P} 1-\mathrm{C} 1-\mathrm{C} 2-\mathrm{C} 3$ & $-176.8(6)$ & $\mathrm{C} 47-\mathrm{C} 48-\mathrm{C} 49-\mathrm{C} 50$ & $3(4)$ \\
\hline $\mathrm{C} 1-\mathrm{C} 2-\mathrm{C} 3-\mathrm{C} 4$ & $-1.2(13)$ & $\mathrm{C} 46-\mathrm{C} 45-\mathrm{C} 50-\mathrm{C} 49$ & $3(3)$ \\
\hline $\mathrm{C} 2-\mathrm{C} 3-\mathrm{C} 4-\mathrm{C} 5$ & $1.3(15)$ & $\mathrm{P} 4-\mathrm{C} 45-\mathrm{C} 50-\mathrm{C} 49$ & $178.2(16)$ \\
\hline $\mathrm{C} 3-\mathrm{C} 4-\mathrm{C} 5-\mathrm{C} 6$ & $-2.0(16)$ & $\mathrm{C} 48-\mathrm{C} 49-\mathrm{C} 50-\mathrm{C} 45$ & $-4(3)$ \\
\hline $\mathrm{C} 2-\mathrm{C} 1-\mathrm{C} 6-\mathrm{C} 5$ & $-2.3(12)$ & $\mathrm{C} 39-\mathrm{P} 4-\mathrm{C} 45 \mathrm{~B}-\mathrm{C} 46 \mathrm{~B}$ & $10.3(15)$ \\
\hline $\mathrm{P} 1-\mathrm{C} 1-\mathrm{C} 6-\mathrm{C} 5$ & $176.0(7)$ & $\mathrm{C} 38-\mathrm{P} 4-\mathrm{C} 45 \mathrm{~B}-\mathrm{C} 46 \mathrm{~B}$ & $120.5(13)$ \\
\hline $\mathrm{C} 4-\mathrm{C} 5-\mathrm{C} 6-\mathrm{C} 1$ & $2.5(16)$ & $\mathrm{Ag} 3-\mathrm{P} 4-\mathrm{C} 45 \mathrm{~B}-\mathrm{C} 46 \mathrm{~B}$ & $-116.7(12)$ \\
\hline $\mathrm{C} 1-\mathrm{P} 1-\mathrm{C} 7-\mathrm{C} 8$ & $-107.6(4)$ & $\mathrm{C} 39-\mathrm{P} 4-\mathrm{C} 45 \mathrm{~B}-\mathrm{C} 50 \mathrm{~B}$ & $-173.9(14)$ \\
\hline $\mathrm{C} 13-\mathrm{P} 1-\mathrm{C} 7-\mathrm{C} 8$ & $145.1(4)$ & $\mathrm{C} 38-\mathrm{P} 4-\mathrm{C} 45 \mathrm{~B}-\mathrm{C} 50 \mathrm{~B}$ & $-63.7(15)$ \\
\hline $\mathrm{Ag} 1-\mathrm{P} 1-\mathrm{C} 7-\mathrm{C} 8$ & $17.8(4)$ & $\mathrm{Ag} 3-\mathrm{P} 4-\mathrm{C} 45 \mathrm{~B}-\mathrm{C} 50 \mathrm{~B}$ & $59.0(16)$ \\
\hline $\mathrm{C} 1-\mathrm{P} 1-\mathrm{C} 7-\mathrm{C} 12$ & $73.3(5)$ & $\mathrm{C} 50 \mathrm{~B}-\mathrm{C} 45 \mathrm{~B}-\mathrm{C} 46 \mathrm{~B}-\mathrm{C} 47 \mathrm{~B}$ & $1.5(17)$ \\
\hline $\mathrm{C} 13-\mathrm{P} 1-\mathrm{C} 7-\mathrm{C} 12$ & $-34.1(5)$ & $\mathrm{P} 4-\mathrm{C} 45 \mathrm{~B}-\mathrm{C} 46 \mathrm{~B}-\mathrm{C} 47 \mathrm{~B}$ & $177.4(13)$ \\
\hline $\mathrm{Ag} 1-\mathrm{P} 1-\mathrm{C} 7-\mathrm{C} 12$ & $-161.3(4)$ & $\mathrm{C} 45 \mathrm{~B}-\mathrm{C} 46 \mathrm{~B}-\mathrm{C} 47 \mathrm{~B}-\mathrm{C} 48 \mathrm{~B}$ & $-1.1(19)$ \\
\hline $\mathrm{C} 12-\mathrm{C} 7-\mathrm{C} 8-\mathrm{C} 9$ & $1.0(8)$ & $\mathrm{C} 46 \mathrm{~B}-\mathrm{C} 47 \mathrm{~B}-\mathrm{C} 48 \mathrm{~B}-\mathrm{C} 49 \mathrm{~B}$ & $3(3)$ \\
\hline $\mathrm{P} 1-\mathrm{C} 7-\mathrm{C} 8-\mathrm{C} 9$ & $-178.2(5)$ & $\mathrm{C} 47 \mathrm{~B}-\mathrm{C} 48 \mathrm{~B}-\mathrm{C} 49 \mathrm{~B}-\mathrm{C} 50 \mathrm{~B}$ & $-5(3)$ \\
\hline $\mathrm{C} 7-\mathrm{C} 8-\mathrm{C} 9-\mathrm{C} 10$ & $-0.7(9)$ & $\mathrm{C} 46 \mathrm{~B}-\mathrm{C} 45 \mathrm{~B}-\mathrm{C} 50 \mathrm{~B}-\mathrm{C} 49 \mathrm{~B}$ & $-4(2)$ \\
\hline $\mathrm{C} 8-\mathrm{C} 9-\mathrm{C} 10-\mathrm{C} 11$ & $-0.1(10)$ & $\mathrm{P} 4-\mathrm{C} 45 \mathrm{~B}-\mathrm{C} 50 \mathrm{~B}-\mathrm{C} 49 \mathrm{~B}$ & $-179.8(14)$ \\
\hline
\end{tabular}




\begin{tabular}{|c|c|c|c|}
\hline $\mathrm{C} 9-\mathrm{C} 10-\mathrm{C} 11-\mathrm{C} 12$ & $0.7(10)$ & $\mathrm{C} 48 \mathrm{~B}-\mathrm{C} 49 \mathrm{~B}-\mathrm{C} 50 \mathrm{~B}-\mathrm{C} 45 \mathrm{~B}$ & $5(3)$ \\
\hline $\mathrm{C} 10-\mathrm{C} 11-\mathrm{C} 12-\mathrm{C} 7$ & $-0.4(9)$ & $\mathrm{C} 57-\mathrm{P} 5-\mathrm{C} 51-\mathrm{C} 52$ & $138.9(17)$ \\
\hline $\mathrm{C} 8-\mathrm{C} 7-\mathrm{C} 12-\mathrm{C} 11$ & $-0.4(8)$ & $\mathrm{C} 63-\mathrm{P} 5-\mathrm{C} 51-\mathrm{C} 52$ & $28(2)$ \\
\hline $\mathrm{P} 1-\mathrm{C} 7-\mathrm{C} 12-\mathrm{C} 11$ & $178.7(4)$ & $\mathrm{Ag} 3-\mathrm{P} 5-\mathrm{C} 51-\mathrm{C} 52$ & $-94.9(18)$ \\
\hline $\mathrm{C} 7-\mathrm{P} 1-\mathrm{C} 13-\mathrm{P} 2$ & $-79.3(3)$ & $\mathrm{C} 57-\mathrm{P} 5-\mathrm{C} 51-\mathrm{C} 56$ & $-44(2)$ \\
\hline $\mathrm{C} 1-\mathrm{P} 1-\mathrm{C} 13-\mathrm{P} 2$ & $170.0(3)$ & $\mathrm{C} 63-\mathrm{P} 5-\mathrm{C} 51-\mathrm{C} 56$ & $-155.2(17)$ \\
\hline $\mathrm{Ag} 1-\mathrm{P} 1-\mathrm{C} 13-\mathrm{P} 2$ & $50.9(3)$ & $\mathrm{Ag} 3-\mathrm{P} 5-\mathrm{C} 51-\mathrm{C} 56$ & $81.9(19)$ \\
\hline $\mathrm{P} 1-\mathrm{C} 13-\mathrm{P} 2-\mathrm{C} 14$ & $81.1(3)$ & $\mathrm{C} 56-\mathrm{C} 51-\mathrm{C} 52-\mathrm{C} 53$ & $2.3(19)$ \\
\hline $\mathrm{P} 1-\mathrm{C} 13-\mathrm{P} 2-\mathrm{C} 20$ & $-167.4(3)$ & $\mathrm{P} 5-\mathrm{C} 51-\mathrm{C} 52-\mathrm{C} 53$ & $179(2)$ \\
\hline $\mathrm{P} 1-\mathrm{C} 13-\mathrm{P} 2-\mathrm{Ag} 2$ & $-45.8(3)$ & $\mathrm{C} 51-\mathrm{C} 52-\mathrm{C} 53-\mathrm{C} 54$ & $-0.3(18)$ \\
\hline $\mathrm{C} 20-\mathrm{P} 2-\mathrm{C} 14-\mathrm{C} 19$ & $-27.0(5)$ & $\mathrm{C} 52-\mathrm{C} 53-\mathrm{C} 54-\mathrm{C} 55$ & $-4(3)$ \\
\hline $\mathrm{C} 13-\mathrm{P} 2-\mathrm{C} 14-\mathrm{C} 19$ & $79.9(5)$ & $\mathrm{C} 53-\mathrm{C} 54-\mathrm{C} 55-\mathrm{C} 56$ & $7(3)$ \\
\hline $\mathrm{Ag} 2-\mathrm{P} 2-\mathrm{C} 14-\mathrm{C} 19$ & $-153.7(4)$ & $\mathrm{C} 54-\mathrm{C} 55-\mathrm{C} 56-\mathrm{C} 51$ & $-5(3)$ \\
\hline $\mathrm{C} 20-\mathrm{P} 2-\mathrm{C} 14-\mathrm{C} 15$ & $154.7(4)$ & $\mathrm{C} 52-\mathrm{C} 51-\mathrm{C} 56-\mathrm{C} 55$ & $0(3)$ \\
\hline $\mathrm{C} 13-\mathrm{P} 2-\mathrm{C} 14-\mathrm{C} 15$ & $-98.5(4)$ & $\mathrm{P} 5-\mathrm{C} 51-\mathrm{C} 56-\mathrm{C} 55$ & $-177.0(16)$ \\
\hline $\mathrm{Ag} 2-\mathrm{P} 2-\mathrm{C} 14-\mathrm{C} 15$ & $28.0(4)$ & $\mathrm{C} 57-\mathrm{P} 5-\mathrm{C} 51 \mathrm{~B}-\mathrm{C} 52 \mathrm{~B}$ & $159.8(11)$ \\
\hline $\mathrm{C} 19-\mathrm{C} 14-\mathrm{C} 15-\mathrm{C} 16$ & $-2.6(8)$ & $\mathrm{C} 63-\mathrm{P} 5-\mathrm{C} 51 \mathrm{~B}-\mathrm{C} 52 \mathrm{~B}$ & $50.5(12)$ \\
\hline $\mathrm{P} 2-\mathrm{C} 14-\mathrm{C} 15-\mathrm{C} 16$ & $175.8(4)$ & $\mathrm{Ag} 3-\mathrm{P} 5-\mathrm{C} 51 \mathrm{~B}-\mathrm{C} 52 \mathrm{~B}$ & $-71.7(12)$ \\
\hline $\mathrm{C} 14-\mathrm{C} 15-\mathrm{C} 16-\mathrm{C} 17$ & $2.2(9)$ & $\mathrm{C} 57-\mathrm{P} 5-\mathrm{C} 51 \mathrm{~B}-\mathrm{C} 56 \mathrm{~B}$ & $-20.7(16)$ \\
\hline $\mathrm{C} 15-\mathrm{C} 16-\mathrm{C} 17-\mathrm{C} 18$ & $-0.4(10)$ & $\mathrm{C} 63-\mathrm{P} 5-\mathrm{C} 51 \mathrm{~B}-\mathrm{C} 56 \mathrm{~B}$ & $-130.0(14)$ \\
\hline $\mathrm{C} 16-\mathrm{C} 17-\mathrm{C} 18-\mathrm{C} 19$ & $-1.0(11)$ & $\mathrm{Ag} 3-\mathrm{P} 5-\mathrm{C} 51 \mathrm{~B}-\mathrm{C} 56 \mathrm{~B}$ & $107.9(14)$ \\
\hline $\mathrm{C} 17-\mathrm{C} 18-\mathrm{C} 19-\mathrm{C} 14$ & $0.5(10)$ & $\mathrm{C} 56 \mathrm{~B}-\mathrm{C} 51 \mathrm{~B}-\mathrm{C} 52 \mathrm{~B}-\mathrm{C} 53 \mathrm{~B}$ & $-1.0(16)$ \\
\hline $\mathrm{C} 15-\mathrm{C} 14-\mathrm{C} 19-\mathrm{C} 18$ & $1.3(8)$ & $\mathrm{P} 5-\mathrm{C} 51 \mathrm{~B}-\mathrm{C} 52 \mathrm{~B}-\mathrm{C} 53 \mathrm{~B}$ & $178.6(13)$ \\
\hline $\mathrm{P} 2-\mathrm{C} 14-\mathrm{C} 19-\mathrm{C} 18$ & $-177.0(5)$ & $\mathrm{C} 51 \mathrm{~B}-\mathrm{C} 52 \mathrm{~B}-\mathrm{C} 53 \mathrm{~B}-\mathrm{C} 54 \mathrm{~B}$ & $0.0(15)$ \\
\hline $\mathrm{C} 14-\mathrm{P} 2-\mathrm{C} 20-\mathrm{C} 21$ & $77.9(5)$ & $\mathrm{C} 52 \mathrm{~B}-\mathrm{C} 53 \mathrm{~B}-\mathrm{C} 54 \mathrm{~B}-\mathrm{C} 55 \mathrm{~B}$ & $0(3)$ \\
\hline $\mathrm{C} 13-\mathrm{P} 2-\mathrm{C} 20-\mathrm{C} 21$ & $-31.4(6)$ & $\mathrm{C} 53 \mathrm{~B}-\mathrm{C} 54 \mathrm{~B}-\mathrm{C} 55 \mathrm{~B}-\mathrm{C} 56 \mathrm{~B}$ & $2(3)$ \\
\hline $\mathrm{Ag} 2-\mathrm{P} 2-\mathrm{C} 20-\mathrm{C} 21$ & $-154.0(5)$ & $\mathrm{C} 52 \mathrm{~B}-\mathrm{C} 51 \mathrm{~B}-\mathrm{C} 56 \mathrm{~B}-\mathrm{C} 55 \mathrm{~B}$ & $2(2)$ \\
\hline $\mathrm{C} 14-\mathrm{P} 2-\mathrm{C} 20-\mathrm{C} 25$ & $-107.5(5)$ & $\mathrm{P} 5-\mathrm{C} 51 \mathrm{~B}-\mathrm{C} 56 \mathrm{~B}-\mathrm{C} 55 \mathrm{~B}$ & $-177.3(13)$ \\
\hline $\mathrm{C} 13-\mathrm{P} 2-\mathrm{C} 20-\mathrm{C} 25$ & $143.2(5)$ & $\mathrm{C} 54 \mathrm{~B}-\mathrm{C} 55 \mathrm{~B}-\mathrm{C} 56 \mathrm{~B}-\mathrm{C} 51 \mathrm{~B}$ & $-3(3)$ \\
\hline $\mathrm{Ag} 2-\mathrm{P} 2-\mathrm{C} 20-\mathrm{C} 25$ & $20.6(5)$ & $\mathrm{C} 51 \mathrm{~B}-\mathrm{P} 5-\mathrm{C} 57-\mathrm{C} 58$ & $122.4(8)$ \\
\hline $\mathrm{C} 25-\mathrm{C} 20-\mathrm{C} 21-\mathrm{C} 22$ & $-1.3(10)$ & $\mathrm{C} 63-\mathrm{P} 5-\mathrm{C} 57-\mathrm{C} 58$ & $-132.7(5)$ \\
\hline $\mathrm{P} 2-\mathrm{C} 20-\mathrm{C} 21-\mathrm{C} 22$ & $173.2(5)$ & $\mathrm{C} 51-\mathrm{P} 5-\mathrm{C} 57-\mathrm{C} 58$ & $117.1(9)$ \\
\hline $\mathrm{C} 20-\mathrm{C} 21-\mathrm{C} 22-\mathrm{C} 23$ & $-0.9(11)$ & Ag3-P5-C57-C58 & $-2.7(6)$ \\
\hline $\mathrm{C} 21-\mathrm{C} 22-\mathrm{C} 23-\mathrm{C} 24$ & $1.5(12)$ & $\mathrm{C} 51 \mathrm{~B}-\mathrm{P} 5-\mathrm{C} 57-\mathrm{C} 62$ & $-55.6(9)$ \\
\hline $\mathrm{C} 22-\mathrm{C} 23-\mathrm{C} 24-\mathrm{C} 25$ & $0.2(12)$ & $\mathrm{C} 63-\mathrm{P} 5-\mathrm{C} 57-\mathrm{C} 62$ & $49.3(7)$ \\
\hline $\mathrm{C} 21-\mathrm{C} 20-\mathrm{C} 25-\mathrm{C} 24$ & $3.0(9)$ & $\mathrm{C} 51-\mathrm{P} 5-\mathrm{C} 57-\mathrm{C} 62$ & $-60.9(10)$ \\
\hline $\mathrm{P} 2-\mathrm{C} 20-\mathrm{C} 25-\mathrm{C} 24$ & $-171.8(5)$ & $\mathrm{Ag} 3-\mathrm{P} 5-\mathrm{C} 57-\mathrm{C} 62$ & $179.2(6)$ \\
\hline $\mathrm{C} 23-\mathrm{C} 24-\mathrm{C} 25-\mathrm{C} 20$ & $-2.5(11)$ & $\mathrm{C} 62-\mathrm{C} 57-\mathrm{C} 58-\mathrm{C} 59$ & $3.6(11)$ \\
\hline $\mathrm{C} 32-\mathrm{P} 3-\mathrm{C} 26-\mathrm{C} 27$ & $-99.9(4)$ & $\mathrm{P} 5-\mathrm{C} 57-\mathrm{C} 58-\mathrm{C} 59$ & $-174.6(7)$ \\
\hline $\mathrm{C} 38-\mathrm{P} 3-\mathrm{C} 26-\mathrm{C} 27$ & $149.8(4)$ & $\mathrm{C} 57-\mathrm{C} 58-\mathrm{C} 59-\mathrm{C} 60$ & $0.7(14)$ \\
\hline $\mathrm{Ag} 2-\mathrm{P} 3-\mathrm{C} 26-\mathrm{C} 27$ & $23.0(4)$ & $\mathrm{C} 58-\mathrm{C} 59-\mathrm{C} 60-\mathrm{C} 61$ & $-4.4(16)$ \\
\hline $\mathrm{C} 32-\mathrm{P} 3-\mathrm{C} 26-\mathrm{C} 31$ & $79.3(4)$ & $\mathrm{C} 59-\mathrm{C} 60-\mathrm{C} 61-\mathrm{C} 62$ & $3.9(17)$ \\
\hline $\mathrm{C} 38-\mathrm{P} 3-\mathrm{C} 26-\mathrm{C} 31$ & $-31.1(4)$ & $\mathrm{C} 60-\mathrm{C} 61-\mathrm{C} 62-\mathrm{C} 57$ & $0.5(16)$ \\
\hline $\mathrm{Ag} 2-\mathrm{P} 3-\mathrm{C} 26-\mathrm{C} 31$ & $-157.8(4)$ & $\mathrm{C} 58-\mathrm{C} 57-\mathrm{C} 62-\mathrm{C} 61$ & $-4.1(13)$ \\
\hline $\mathrm{C} 31-\mathrm{C} 26-\mathrm{C} 27-\mathrm{C} 28$ & $-1.0(7)$ & $\mathrm{P} 5-\mathrm{C} 57-\mathrm{C} 62-\mathrm{C} 61$ & $173.9(7)$ \\
\hline $\mathrm{P} 3-\mathrm{C} 26-\mathrm{C} 27-\mathrm{C} 28$ & $178.2(4)$ & $\mathrm{C} 51 \mathrm{~B}-\mathrm{P} 5-\mathrm{C} 63-\mathrm{P} 6$ & $-173.4(6)$ \\
\hline $\mathrm{C} 26-\mathrm{C} 27-\mathrm{C} 28-\mathrm{C} 29$ & $1.3(8)$ & $\mathrm{C} 57-\mathrm{P} 5-\mathrm{C} 63-\mathrm{P} 6$ & $79.5(3)$ \\
\hline $\mathrm{C} 27-\mathrm{C} 28-\mathrm{C} 29-\mathrm{C} 30$ & $-1.0(8)$ & $\mathrm{C} 51-\mathrm{P} 5-\mathrm{C} 63-\mathrm{P} 6$ & $-171.6(8)$ \\
\hline
\end{tabular}




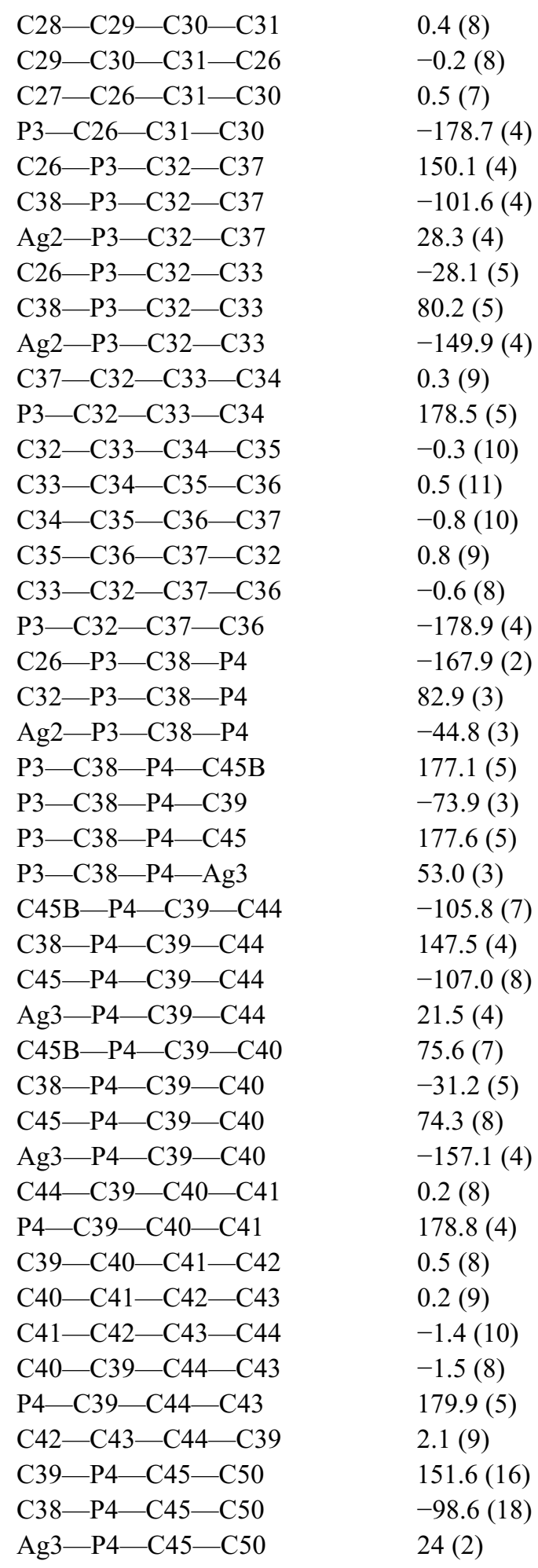

$-0.2(8)$

$0.5(7)$

$-178.7(4)$

150.1 (4)

$-101.6(4)$

28.3 (4)

$-28.1(5)$

$80.2(5)$

$-149.9(4)$

$0.3(9)$

$178.5(5)$

$-0.3(10)$

$0.5(11)$

$-0.8(10)$

$0.8(9)$

$-0.6(8)$

$-178.9(4)$

$-167.9(2)$

82.9 (3)

-44.8 (3)

177.1 (5)

$-73.9(3)$

$177.6(5)$

$53.0(3)$

$-105.8(7)$

$147.5(4)$

$-107.0(8)$

$21.5(4)$

$75.6(7)$

$-31.2(5)$

$74.3(8)$

$-157.1(4)$

$0.2(8)$

$178.8(4)$

$0.5(8)$

$0.2(9)$

$-1.4(10)$

$-1.5(8)$

$179.9(5)$

$2.1(9)$

$151.6(16)$

$-98.6(18)$

24 (2)

\begin{tabular}{|c|c|}
\hline $\mathrm{Ag} 3-\mathrm{P} 5-\mathrm{C} 63-\mathrm{P} 6$ & $-52.6(3)$ \\
\hline $\mathrm{P} 5-\mathrm{C} 63-\mathrm{P} 6-\mathrm{C} 64$ & $-81.0(3)$ \\
\hline $\mathrm{P} 5-\mathrm{C} 63-\mathrm{P} 6-\mathrm{C} 70$ & $171.4(3)$ \\
\hline $\mathrm{P} 5-\mathrm{C} 63-\mathrm{P} 6-\mathrm{Ag} 1$ & $54.5(3)$ \\
\hline $\mathrm{C} 70-\mathrm{P} 6-\mathrm{C} 64-\mathrm{C} 65$ & $-132.3(5)$ \\
\hline $\mathrm{C} 63-\mathrm{P} 6-\mathrm{C} 64-\mathrm{C} 65$ & $117.6(5)$ \\
\hline $\mathrm{Ag} 1-\mathrm{P} 6-\mathrm{C} 64-\mathrm{C} 65$ & $-12.5(5)$ \\
\hline $\mathrm{C} 70-\mathrm{P} 6-\mathrm{C} 64-\mathrm{C} 69$ & $44.6(5)$ \\
\hline $\mathrm{C} 63-\mathrm{P} 6-\mathrm{C} 64-\mathrm{C} 69$ & $-65.5(5)$ \\
\hline $\mathrm{Ag} 1-\mathrm{P} 6-\mathrm{C} 64-\mathrm{C} 69$ & $164.4(4)$ \\
\hline $\mathrm{C} 69-\mathrm{C} 64-\mathrm{C} 65-\mathrm{C} 66$ & $0.0(9)$ \\
\hline $\mathrm{P} 6-\mathrm{C} 64-\mathrm{C} 65-\mathrm{C} 66$ & $177.0(5)$ \\
\hline $\mathrm{C} 64-\mathrm{C} 65-\mathrm{C} 66-\mathrm{C} 67$ & $-0.3(11)$ \\
\hline $\mathrm{C} 65-\mathrm{C} 66-\mathrm{C} 67-\mathrm{C} 68$ & $1.1(13)$ \\
\hline $\mathrm{C} 66-\mathrm{C} 67-\mathrm{C} 68-\mathrm{C} 69$ & $-1.7(12)$ \\
\hline $\mathrm{C} 67-\mathrm{C} 68-\mathrm{C} 69-\mathrm{C} 64$ & $1.5(11)$ \\
\hline $\mathrm{C} 65-\mathrm{C} 64-\mathrm{C} 69-\mathrm{C} 68$ & $-0.6(10)$ \\
\hline $\mathrm{P} 6-\mathrm{C} 64-\mathrm{C} 69-\mathrm{C} 68$ & $-177.6(5)$ \\
\hline $\mathrm{C} 64-\mathrm{P} 6-\mathrm{C} 70-\mathrm{C} 71$ & $-137.1(5)$ \\
\hline $\mathrm{C} 63-\mathrm{P} 6-\mathrm{C} 70-\mathrm{C} 71$ & $-27.6(5)$ \\
\hline $\mathrm{Ag} 1-\mathrm{P} 6-\mathrm{C} 70-\mathrm{C} 71$ & $93.0(5)$ \\
\hline $\mathrm{C} 64-\mathrm{P} 6-\mathrm{C} 70-\mathrm{C} 75$ & $50.0(4)$ \\
\hline $\mathrm{C} 63-\mathrm{P} 6-\mathrm{C} 70-\mathrm{C} 75$ & $159.5(4)$ \\
\hline $\mathrm{Ag} 1-\mathrm{P} 6-\mathrm{C} 70-\mathrm{C} 75$ & $-79.9(4)$ \\
\hline $\mathrm{C} 75-\mathrm{C} 70-\mathrm{C} 71-\mathrm{C} 72$ & $-0.6(9)$ \\
\hline $\mathrm{P} 6-\mathrm{C} 70-\mathrm{C} 71-\mathrm{C} 72$ & $-173.3(5)$ \\
\hline $\mathrm{C} 70-\mathrm{C} 71-\mathrm{C} 72-\mathrm{C} 73$ & $-0.1(11)$ \\
\hline $\mathrm{C} 71-\mathrm{C} 72-\mathrm{C} 73-\mathrm{C} 74$ & $-0.1(11)$ \\
\hline $\mathrm{C} 72-\mathrm{C} 73-\mathrm{C} 74-\mathrm{C} 75$ & $0.9(11)$ \\
\hline $\mathrm{C} 71-\mathrm{C} 70-\mathrm{C} 75-\mathrm{C} 74$ & $1.4(8)$ \\
\hline $\mathrm{P} 6-\mathrm{C} 70-\mathrm{C} 75-\mathrm{C} 74$ & $174.7(5)$ \\
\hline $\mathrm{C} 73-\mathrm{C} 74-\mathrm{C} 75-\mathrm{C} 70$ & $-1.6(10)$ \\
\hline $\mathrm{C} 77-\mathrm{N} 2-\mathrm{C} 76-\mathrm{N} 1$ & $-176.3(6)$ \\
\hline $\mathrm{C} 77-\mathrm{N} 2-\mathrm{C} 76-\mathrm{S} 1$ & $4.5(11)$ \\
\hline $\mathrm{C} 76-\mathrm{N} 2-\mathrm{C} 77-\mathrm{C} 78$ & $-152.1(7)$ \\
\hline $\mathrm{C} 76-\mathrm{N} 2-\mathrm{C} 77-\mathrm{C} 82$ & $32.6(11)$ \\
\hline $\mathrm{C} 82-\mathrm{C} 77-\mathrm{C} 78-\mathrm{C} 79$ & $-1.6(12)$ \\
\hline $\mathrm{N} 2-\mathrm{C} 77-\mathrm{C} 78-\mathrm{C} 79$ & $-177.2(7)$ \\
\hline $\mathrm{C} 77-\mathrm{C} 78-\mathrm{C} 79-\mathrm{C} 80$ & $0.1(14)$ \\
\hline $\mathrm{C} 78-\mathrm{C} 79-\mathrm{C} 80-\mathrm{C} 81$ & $0.7(15)$ \\
\hline $\mathrm{C} 79-\mathrm{C} 80-\mathrm{C} 81-\mathrm{C} 82$ & $0.0(15)$ \\
\hline $\mathrm{C} 78-\mathrm{C} 77-\mathrm{C} 82-\mathrm{C} 81$ & $2.2(12)$ \\
\hline $\mathrm{N} 2-\mathrm{C} 77-\mathrm{C} 82-\mathrm{C} 81$ & $177.4(7)$ \\
\hline $\mathrm{C} 80-\mathrm{C} 81-\mathrm{C} 82-\mathrm{C} 77$ & $-1.4(14)$ \\
\hline
\end{tabular}

Ag3-P5-C63-P6
$\mathrm{P} 5-\mathrm{C} 63-\mathrm{P} 6-\mathrm{C} 64$

$\mathrm{P} 5-\mathrm{C} 63-\mathrm{P} 6-\mathrm{C} 70$

$\mathrm{C} 70-\mathrm{P} 6-\mathrm{C} 64-\mathrm{C} 65$

C63-P6-C64-C65

Ag1-P6-C64-C65

C70-P6-C64-C69

C63-P6-C64-C69

Ag1-P6-C64-C69

P6-C64-C65-C66

C64-C65-C66-C67

C67-C68-C69-C64

C65-C64-C69- 68

$\mathrm{P} 6-\mathrm{C} 64-\mathrm{C} 69-\mathrm{C} 68$

$\mathrm{C} 64-\mathrm{P} 6-\mathrm{C} 70-\mathrm{C} 71$

$\mathrm{C} 63-\mathrm{P} 6-\mathrm{C} 70-\mathrm{C} 71$

Ag1-P6-C70-C71

C63-P6- $770-\mathrm{C} 75$

Ag1-P6-C70-C75

$\mathrm{C} 75-\mathrm{C} 70-\mathrm{C} 71-\mathrm{C} 72$

$\mathrm{C} 70=\mathrm{C} 71-\mathrm{C} 72-\mathrm{C} 73$

$\mathrm{C} 71-\mathrm{C} 72-\mathrm{C} 73-\mathrm{C} 74$

$\mathrm{C} 72-\mathrm{C} 73-\mathrm{C} 74-\mathrm{C} 75$

$\mathrm{C} 71-\mathrm{C} 70-\mathrm{C} 75-\mathrm{C} 74$

$\mathrm{P} 6-\mathrm{C} 70-\mathrm{C} 75-\mathrm{C} 74$

C73-C74-C75-C70

$\mathrm{C} 77-\mathrm{N} 2-\mathrm{C} 76-\mathrm{N} 1$

$\mathrm{C} 77-\mathrm{N} 2-\mathrm{C} 76-\mathrm{S} 1$

$\mathrm{C} 77-\mathrm{C} 78$

$\mathrm{C} 82-\mathrm{C} 77-\mathrm{C} 78-\mathrm{C} 79$

$\mathrm{N} 2-\mathrm{C} 77-\mathrm{C} 78-\mathrm{C} 79$

$\mathrm{C} 78-\mathrm{C} 79-\mathrm{C} 80-\mathrm{C} 81$

$\mathrm{C} 79-\mathrm{C} 80-\mathrm{C} 81-\mathrm{C} 82$

$\mathrm{N} 2-\mathrm{C} 77-\mathrm{C} 82-\mathrm{C} 81$
$\mathrm{C} 80-\mathrm{C} 81-\mathrm{C} 82-\mathrm{C} 77$
$-52.6(3)$
$-81.0(3)$
$171.4(3)$
$54.5(3)$
$-132.3(5)$
$117.6(5)$
$-12.5(5)$
$44.6(5)$
$-65.5(5)$
$164.4(4)$
$0.0(9)$
$177.0(5)$
$-0.3(11)$
$1.1(13)$
$-1.7(12)$
$1.5(11)$
$-0.6(10)$
$-177.6(5)$
$-137.1(5)$
$-27.6(5)$
$93.0(5)$
$50.0(4)$
$159.5(4)$
$-79.9(4)$
$-0.6(9)$
$-173.3(5)$
$-0.1(11)$
$-0.1(11)$
$0.9(11)$
$1.4(8)$
$174.7(5)$
$-1.6(10)$
$-176.3(6)$
$4.5(11)$
$-152.1(7)$
$32.6(11)$
$-1.6(12)$
$-177.2(7)$
$0.1(14)$
$0.7(15)$
$0.0(15)$
$2.2(12)$
$177.4(7)$
$-1.4(14)$
-19 
supporting information

Hydrogen-bond geometry $\left(A,{ }^{\circ}\right)$

\begin{tabular}{lllll}
\hline$D-\mathrm{H} \cdots A$ & $D-\mathrm{H}$ & $\mathrm{H} \cdots A$ & $D \cdots A$ & $D-\mathrm{H} \cdots A$ \\
\hline $\mathrm{N} 1-\mathrm{H} 1 A \cdots \mathrm{I} 3$ & 0.86 & 2.82 & $3.633(9)$ & 159 \\
$\mathrm{~N} 2-\mathrm{H} 2 A \cdots \mathrm{I} 3$ & 0.86 & 2.72 & $3.568(6)$ & 170 \\
$\mathrm{C} 53-\mathrm{H} 53 \cdots \mathrm{S} 1^{\mathrm{i}}$ & 0.99 & 2.88 & $3.67(2)$ & 143 \\
$\mathrm{C} 13-\mathrm{H} 13 B \cdots \mathrm{I} 3$ & 0.99 & 3.04 & $3.933(4)$ & 153
\end{tabular}

Symmetry code: (i) $-x+3 / 2, y-1 / 2,-z+3 / 2$. 